\title{
What Lies Beneath - A Survey of Affective Theory Use in Computational Models of Emotion
}

This paper was downloaded from TechRxiv (https://www.techrxiv.org).

\section{LICENSE}

CC BY 4.0

SUBMISSION DATE / POSTED DATE

20-01-2022 / 25-01-2022

\section{CITATION}

Smith, Geneva; Carette, Jacques (2022): What Lies Beneath - A Survey of Affective Theory Use in Computational Models of Emotion. TechRxiv. Preprint. https://doi.org/10.36227/techrxiv.18779315.v1

$\mathrm{DOI}$ 


\title{
What Lies Beneath-A Survey of Affective Theory Use in Computational Models of Emotion
}

\author{
Geneva M. Smith ๑ and Jacques Carette $\odot$
}

\begin{abstract}
Affective computing encompasses the research and development of systems that can recognize, express, and "have" emotions. Its literature is already vast, which is a hindrance for newcomers. Those who wish to create Computational Models of Emotion (CMEs) must first identify what kind of system they want to build, then identify affective theories that match its requirements. This survey aims to help designers of CMEs that generate emotions in computer agents and user interfaces with this latter task. We give an overview of $63 \mathrm{CMEs}$ from different domains, and identify which affective theories they use and why. Some of these CMEs also use affective theories to express emotion and for other design purposes. We also analyse these instances to better understand the complete system. The survey closes with a brief summary of how CMEs generally use each encountered theory. The survey is meant as a guideline for deciding which affective theories to use for new CME designs that generate emotions.
\end{abstract}

Index Terms-Affective computing, artificial intelligence, computational models of emotion (CME), psychology.

\section{INTRODUCTION}

A FFECTIVE Computing introduces emotion as a concern in programs so that they may recognize and respond to human users more intelligently [1, pp. 3, 50]. There are three main affective computing tasks [2, pp. 4], [3, pp. 2] that enable this human-centered approach:

- Emotion Recognition, to capture user information like speech and gesture to infer their current affective state,

- Emotion Generation, to produce an affective state given the current program and environment state, and

- Emotion Effects on Behavior, to change a program's behavior (e.g. facial expressions, gestures, or movements) given its affective state.

Infrequently another task, Emotion Effects on Cognitive Processes or Cognitive Consequences of Emotions [4, pp. 100] is added. We focus on emotion generation and some aspects of emotion effects on behavior.

\subsection{Computational Models of Emotion}

A Computational Model of Emotion (CME) is a software system whose design is influenced by affective research [5, pp. 2, 14]. CMEs use at least one affective theory as the basis for its stimuli evaluation, emotion elicitation, and emotional behavior generation mechanisms. A critical step in CME design is choosing affective theories that best suit the design requirements. This is a difficult task. Each theory has their own assumptions on how different affective components integrate, how emotions are differentiated, and on what stage of the emotion process they focus on [2, pp. 10-11].

Given the large number of affective theories available (we've seen at least 27), it is unrealistic for developers of new systems to attempt to understand them all. A good

- The authors are with the G-ScalE Lab in the Department of Computing and Software, McMaster University, 1280 Main St. West, Hamilton, ON, L8S 4L8, Canada. E-mail: smithgm@mcmaster.ca, carette@mcmaster.ca

Manuscript received December 8, 2021. (Corresponding author: G. M. Smith) Digital Object Identifier no. 10.36227/techrxiv.18779315 first step is to compare a categorization of the theories that generally organize them into families, which relate the theories by core assumptions or focus [2, pp. 11, 20]. From there, one can identify a subset of theories to analyze to see if they satisfy the design requirements, their level of empirical validation, and how they might be used together.

This survey explores 63 CMEs from different application domains. Its goal is to provide an overview of affective theories that appear in CME designs, and the reasons for that choice ${ }^{1}$. Seeing affective theories in context has two advantages. First, CMEs translate theories into concrete computational representations, thus dispelling the fuzziness of the theories' natural language presentations. The second and greater advantage is that a CME targeted at a specific application domain will illustrate the underlying theory's strengths and how it could be mechanized. In practice, designers often combine theories-sometimes implicitly-to achieve the desired CME functionality, because single theories do not address all aspects of emotion or the available empirical data [7, pp. 10]. Thus the role assigned to a theory could be an indicator of its strengths.

\subsection{Survey Scope}

We only include CMEs that include emotion generation. We used snowballing [8] to obtain systems, starting from wellknown ones (e.g. ALMA (38), EMA (26)) and papers like [9], then following references and discovered keywords. Some systems are direct iterations of prior designs-see Table 2 for CME "genealogy". Prior systems are not surveyed unless they are sufficiently different to warrant exploration. Prior systems that are not psychologically grounded are also omitted, though mentioned when important ideas were borrowed from them.

The systems are loosely grouped by application domain (Table 1) to capture the general design intent. These categories are not restrictive-a CME might fit into multiple

1. See [6, pp. 370-372] for some historical context as well. 
TABLE 1

Overview of the Application Domains for Surveyed CMEs that Generate Emotion

\begin{tabular}{|c|c|}
\hline Domain & Systems \\
\hline Multi-Purpose & $\begin{array}{l}\text { 1) Affective Reasoner (AffectR), 2) Cathexis, 3) Emotion Model (EmMod), 4) FLAME, } \\
\text { 5) SCREAM, 6) MAMID, 7) TABASCO, 8) WASABI, 9) Maggie, 10) AKR Scheme, 11) General } \\
\text { Virtual Human (GVH), 12) ParleE, 13) Personality, Mood, and Emotion Space (PMES), } \\
\text { 14) Interdependent Model of Personality, Motivations, Emotion, and Mood (IM-PMEB), } \\
\text { 15) DER, 16) GenIA }{ }^{3} \text {, 17) InFra, 18) FAtiMA Modular (FAtiMA-M), 19) Hybrid Model of } \\
\text { Emotion-Eliciting Conditions (HybridC) }\end{array}$ \\
\hline Natural Language Processing & 20) SOM, 21) Hourglass of Emotions (HoE) \\
\hline Cognitive Architecture & 22) Soar, 23) LIDA, 24) CLARION \\
\hline Affective Research & 25) ACRES, 26) EMA, 27) Will, 28) ELSA, \\
\hline $\begin{array}{l}\text { Military and Emergency Personnel } \\
\text { Training }\end{array}$ & 29) Émile, 30) EMOTION, 31) JBdiEmo, 32) DETT \\
\hline Soft Skills Training & 33) Puppet, 34) CBI, 35) FAtiMA, 36) TARDIS \\
\hline Virtual Assistants and Companions & $\begin{array}{l}\text { 37) Greta, 38) ALMA, 39) Eva, 40) PPAD-Algorithm (PPAD-Algo), 41) PSA Heart, 42) Peedy } \\
\text { the Parrot, 43) ERDAMS, 44) TEATIME, 45) Presence, 46) Fuzzy Finite State Machine } \\
\text { Emotion Simulator (FFSM-Emo) }\end{array}$ \\
\hline Social Robots & $\begin{array}{l}\text { 47) Kismet, 48) KaMERo, 49) Roboceptionist (R-Cept), 50) Partially Observable Markov } \\
\text { Decision Process for Cognitive Appraisal (POMDP-CA), 51) TAME, 52) iPhonoid, } \\
\text { 53) Plutchik's Wheel of Emotions Inspired (PWE-I), 54) GRACE, 55) EEGS }\end{array}$ \\
\hline Art and Entertainment & $\begin{array}{l}\text { 56) Socioemotional State (SocioEmo), 57) The Soul, 58) GAMYGDALA, 59) Mind Module } \\
(\mathrm{MM}), 60) \mathrm{Em} / \mathrm{Oz}, 61) \mathrm{S} 3 \mathrm{~A}, 62) \text { FeelMe, 63) INVITE Game Agents (INVITE-A) }\end{array}$ \\
\hline
\end{tabular}

domains. The purpose is to capture the prevalent intent, which should have guided the design process the most.

- Multi-Purpose CMEs (Systems 1-19) are not limited to one specific task. These systems: explicitly list multiple, sufficiently different potential uses [10, pp. 3-6], [11], [12], [13]; name a general type of CME environment [14], [15], [16], [17], [18], [19], [20], [21], [22, pp. 10], [23], [24], [25, pp. 60]; and allow users to integrate their own affective theory implementations [26, pp. 12-13], [27], [28].

- Natural Language Processing CMEs (Systems 20-21) read, decipher, comprehend, and analyze human language, focusing on affective content [29], [30].

- Cognitive Architectures (Systems 22-24) implement theories concerned with the components of the mind and interactions between them [31], [32], [33].

- Affective Research CMEs (Systems 25-28) explore aspects of affect or affective system design. They are typically stricter about the system's behaviors, as they aim to test an affective theory [34], [35] or replicate observed affective phenomena [36], [37].

- Military and Emergency Personnel Training CMEs (Systems 29-32) help train personnel for emotionallycharged scenarios in consequence free environments [38], [39], [40], or run simulations where affect is a factor [41].

- CMEs for Soft Skills Training (Systems 33-36) help train life skills that can be difficult to hone with traditional techniques. This includes emotional intelligence [42, pp. 153], problem solving under pressure [43], empathy [44], and interview skills [45].

- Virtual Assistants with CMEs (Systems 37-46) have a virtual embodiment, interacting with the user in a conversational capacity. They focus on: believability [46], [47], [48], [49]; improved interface usability [50], [51], [52], [53]; or both [54, pp. 762], [42, pp. 158].

- CMEs for Social Robots (Systems 47-55) are different from virtual assistants because of robot's physical embodiment [55, pp. 120]. These CMEs aim to humanize robots and improve human-robot interactions by adding a social dimension to them [56], [57], [58], [59, pp. 209]—sometimes over extended time frames [55, pp. 122-124], [60], [61] -and to provide companionship [62], [63].

- CMEs for Art and Entertainment (Systems 56-63) are often used for improving agent believability, changing the focus from strict adherence to psychological validity to interesting and entertaining behaviors. However, agent behaviors must remain plausible to be effective [64, p. 216-217]. There are CMEs for: developer tools [65], [66], [67], [68]; agent architectures [69, pp. 3], [70, pp. 31], [71]; and exploring how emotion influences believability [72, pp. 9].

\subsection{Survey Organization}

In Section 2, we extract the theories used and the justification for choosing them from the surveyed CMEs. CMEs perform two main tasks-emotion generation and expressionas well as some others. Correspondingly, we survey:

- Emotion Generation (Table 4): CMEs use affective theories to specify what emotions are possible, their eliciting conditions (EECs), the affective space they occupy, appraisal variables, and the order of process steps. Multiple theories are often combined for this purpose, such as using the emotions from one theory and the appraisal variables of another. Thus we organize CMEs by the kinds of theories they use. We use the broad categories of [4]-discrete, dimensional, appraisal, and neurobiological-but see [73, pp. 280] for a finer-grained categorization. When a CME can be programmed with a user's choice of theories, we examine its default implementation.

- Emotion Expression (Table 5): As this is often considered distinct from generation, we examine affective theories 
selected for expression separately. CMEs that do both generation and expression might use separate theories for each task, or the same combination of theories for both.

- Other Uses (Table 6): Some CMEs do not explicitly model an affective theory, but it still plays a significant role in its design. Examining their role in the design process helps reveal the rationale behind a CME's design.

In Section 3 we examine the theories (Table 3) that appear in CMEs at least five times to understand their strengths. This could reveal convergence on their use across systems. Notes are also made about which theories have been combined in CMEs. Throughout, we use abbreviations for CME and affective theory names-some of them our own-to increase the legibility of the text.

\section{SuRVEY}

CMEs often use a theory for multiple purposes. We organize CMEs by theory to examine all uses of a theory simultaneously, which allows us to deduce reasons for the overlap. We cover each category in turn-discrete in Section 2.1, dimensional in Section 2.2, appraisal in Section 2.3, and neurobiological in Section 2.4. Within a section, the ordering of theories is chosen to make the narrative smoother.

\subsection{Discrete Theories}

Discrete or categorical theories emphasize a small set of emotions, often called basic or primary emotions, that have evolved via natural selection [140, pp. 305], [73, pp. 280], [6, pp. 352].

\subsubsection{Izard (Systems 2, 15, 47)}

Izard (Iz.) appears in CMEs to define facial expressions and the functional role of each emotion. However, it is not used alone-or, arguably, primarily-for these tasks. Iz. is combined with Ek. to define emotion categories based on facial expressions [15, pp. 71], [26, pp. 4-5], [20, pp. 142-143]. This is a "safe" combination, as Iz. shares the assumption that facial expressions are a fundamental component of emotion [141, pp. 83, 85-92]. There is also overlap in the facial expression prototypes (e.g. Distress-Anguish), strengthening their shared foundations on this aspect. Iz. is combined with Plu. to explain emotion "mixtures" [25, pp. 63] and the functional role of emotions [55, pp. 129], which are both included in Iz.'s theory [141, pp. 64-65, 83, 97].

The infrequent use of Iz. might be due to its focus on affective development, such as how it contributes to personality and social development [108, pp. 847], [110, pp. 253254]. It presents emotion as a subsystem of personality that also includes perception, cognition, motor systems, and a network of interrelated systems that act automatically and unconsciously [141, pp. 17, 44]. This suggests untapped potential. Iz. could be useful for CMEs that want to tie personality to emotions without creating a personality module (e.g. variable emotion thresholds), and to define an emotiondriven learning system for personality development [141, pp. 17, 45]. Iz. also connects drives to the larger personality system [141, pp. 44], which could be useful for CMEs that include them. Cathexis (2) uses Iz. to differentiate between cognitive and non-cognitive emotion elicitors [15, pp. 71]. This suggest that Iz. would also be useful for separately triggering CME's reactive and deliberative systems.

2.1.2 Ekman (Systems 2-3, 5, 8, 10-13, 15, 19, 33, 37, 47, $49,51-52,57,59)$

CMEs use Ekman (Ek.) when they need to define or allow the assignment of facial expressions for specific emotions [16, pp. 66], [18, pp. 594], [46, pp. 91], [55, pp. 129, 140], [21, pp. 253, 257], [66, pp. 343], [97, pp. 740], which is indeed its focus [111, pp. 1]. Some CMEs have played it safe, listing Ek.'s emotions as outputs of the emotion generation process to ensure a direct connection between generation and expression [42, pp. 155], [22, pp. 84, 100], [19, pp. 109].

While dimensional models may produce more accurate expressions, discrete categories are easier to implement [61, pp. 324]. FACS [112] is particularly useful for these CME needs, as they break down the face into individual muscles and show how they can combine into expressions [20, pp. 146], [66, pp. 343], allowing expressions to be synthesized both discretely and continuously [63, pp. 217]. Facial muscles are well-documented as part of human muscle anatomy, making it possible to mechanize them in a realistic way [46, pp. 87-89]. This is essential for CMEs where users expect affective expressions [63, pp. 211].

Ek. has a small list of emotion/expression pairs, which can be limiting, but still compelling due to their hypothesized universality [42, pp. 155], [69, pp. 8]. However, there might be more [22, pp. 18-19]. ParleE (12) suggests that other pairs can be added given sufficient evidence, motivating its combination of Ek. and Iz. [20, pp. 142-143]. Iz. helps with this task [55, pp. 129], [26, pp. 3-4] as it shares similar views and also has a system for identifying facial expressions, although its primary focus on infants and young children [142]. Both Ekman and Izard unknowingly performed the same cross-cultural studies at the same time under Tomkins [111, pp. 3], which could explain the shared views. They found comparable results, though Izard found more expressions than Ekman. This eases their combination when choosing categories for emotion generation based on facial expressions and "universality" [15, pp. 71].

CMEs have also used Ek. for emotion expression without using Ek.'s specific categories by using subsets of emotions from other theories in emotion generation-such as OCC [16, pp. 65-66], [46, pp. 91, 94] or a dimensional model [55, pp. 129, 140], [66, pp. 343] —or by simply not connecting all possible emotions to facial expressions [20, pp. 142]. O \& JL is another appraisal theory that CMEs use that can connect to Ek. [25, pp. 63], [15, pp. 71], as there is explicit overlap in their primary emotion list [132, pp. 209, 217].

SCREAM (5) is the only CME surveyed that uses Ek.'s display rules [111, pp. 4] to control emotional displays given a social and interaction context [14, pp. 231-232]. Iz. also suggests that display rules can explain some social and cultural differences in emotion expressions [141, pp. 84-85]. This makes Ek. a strong candidate-if not the de facto standard [7, pp. 4]-for CMEs with facial expression-centered emotion expression components. 
TABLE 2

Overview of the Contributing Designs of CMEs

\begin{tabular}{|c|c|c|}
\hline & System & Builds On \\
\hline 5 & SCREAM & AffectR (1), Em/Oz (60) ${ }^{2}$ \\
\hline 7 & TABASCO & $3 \mathrm{~T}[74]^{1}$ \\
\hline 8 & WASABI & $\operatorname{MAX}[75]^{3}$ \\
\hline 10 & AKR & Will $(27)^{1}$, GOLEM $[76]^{1}$ \\
\hline 11 & GVH & Autonomous Virtual Human Dialog System $[77]^{3}$ \\
\hline 12 & ParleE & Cathexis $(2)^{1}$, FLAME (4), Émile $(29)^{1,2}$, Em/Oz (60) \\
\hline 13 & PMES & ALMA (38) \\
\hline 14 & IM-PMEB & ALMA (38) \\
\hline 16 & GenIA $^{3}$ & EMA (26), ALMA (38), ERDAMS (43), O3A [78 $]^{3}$, AgentSpeak [79] ${ }^{1}$ \\
\hline 17 & InFra & FLAME (4) \\
\hline 18 & FAtiMA-M & FAtiMA $(35)^{3}$, ORIENT [80] $]^{1}$, Computational Appraisal Architecture [9, pp. 31] \\
\hline 19 & HybridC & EMIA $[81]^{3,4}$ \\
\hline 22 & Soar & $\mathrm{Em} / \mathrm{Oz}(60)^{2}, \mathrm{PEACTIDM}[82]^{1}$ \\
\hline 23 & LIDA & Computational Appraisal Architecture [9, pp. 31] \\
\hline 26 & EMA & AffectR (1), Soar $(22)^{1}$, Will (27) ${ }^{1}$, Émile (29) \\
\hline 27 & Will & ACRES $(25)^{3}$ \\
\hline 29 & Émile & AffectR (1), Cathexis $(2)^{2}$, Em/Oz (60), NML1 $[83]^{1}$, Steve $[84]^{5}$, Affect Editor $[85]^{5}$ \\
\hline 30 & EMOTION & GVH (11): Generic Model [86] \\
\hline 31 & JBdiEmo & Jadex $[87]^{1}$ \\
\hline 32 & DETT & MANA $[88]^{1,2}$ \\
\hline 33 & Puppet & S3A $(61)^{1}$ \\
\hline 35 & FAtiMA & $\operatorname{TABASCO}(7)^{1}$, EMA (26), CBI (34), S3A (61), FearNot! [89] ${ }^{3}$ \\
\hline 36 & TARDIS & Greta $(37)^{5}$, ALMA $(38)^{1}$, SocioEmo $(56)^{1}$ \\
\hline 38 & ALMA & EmotionEngine [90], [91] ${ }^{3}$ \\
\hline 39 & Eva & $\operatorname{ALMA}(38)^{1}$ \\
\hline 40 & PPAD-Algo & ALMA (38), Eva (39) ${ }^{1}$ \\
\hline 43 & ERDAMS & $\begin{array}{l}\text { AffectR (1), ParleE (12) }{ }^{2}, \text { DER (15), Émile (29)/“Jack and Steve" }{ }^{2}, \text { Em/Oz (60), Corpora } \\
\text { Coding [92] }\end{array}$ \\
\hline 45 & Presence & PPP $[93]^{1,3}$ \\
\hline 47 & Kismet & Cathexis $(2)^{3}$ \\
\hline 48 & KaMERo & Steward Robot $[94]^{3}$, Sentiment Relation Model $[95]^{3}$ \\
\hline 49 & R-Cept & Vickia $[96]^{1,5}$ \\
\hline 52 & iPhonoid & Interactive Robot System with Memory $[97]^{3}$, AEIS $[98]^{1}$ \\
\hline 53 & PWE-I & HED [99 $]^{1}$, Mood Prediction $[100]^{1}$ \\
\hline 54 & GRACE & EmotiRob $[101]^{3}$ \\
\hline 55 & EEGS & Computational Appraisal Architecture [9, pp. 31] \\
\hline 56 & SocioEmo & ParleE $(12)^{1}$, Émile (29), ALMA $(38)^{1}$, Em/Oz (60), E/P Model [102] ${ }^{3}$ \\
\hline 57 & The Soul & $\operatorname{ALMA}(38)^{1}$, Animating Expressions $[103]^{3}$ \\
\hline 58 & GAMYGDALA & $\mathrm{Em} / \mathrm{Oz}(60)^{2}$ \\
\hline 59 & MM & Will $(27)^{1}$ \\
\hline 60 & $\mathrm{Em} / \mathrm{Oz}$ & Tok $[104]^{1}$, Hap $[105]^{1}$ \\
\hline 61 & S3A & Will $(27)^{4}, \mathrm{Em} / \mathrm{Oz}(60)$ \\
\hline 63 & INVITE-A & INVITE Game [106] ${ }^{3}$, Grudge-holding/Grateful Agent Scenarios [107] \\
\hline
\end{tabular}

${ }^{1}$ For domain specific agent capabilities that do not explicitly model agent emotion, influence emotion via other kinds of affect, map emotion to another affect type, or are implementation-specific.

${ }^{2}$ For domain specific agent capabilities that are affective in nature, but have unclear theoretical roots.

${ }^{3}$ Direct or close descendant of this system.

${ }^{4}$ The relationship is inferred from chosen affective theories and model definitions [25, pp. 61], [71, pp. 37, 48].

${ }^{5}$ For agent embodiment only.

\subsubsection{Plutchik (Systems 8, 17, 19-21, 46-47, 53)}

Plutchik (Plu.) tends to appear in CMEs for its structure, ability to mix emotions, and/or its connection between emotions and adaptational behaviors [114, pp. 203-204].

Plu.'s circumplex structure, featuring an intensity di- mension, affords both the understandability of emotion categories and the computational ease of dimensional theories. This allows CMEs to bridge the gap between machine and human-friendly representations [53, pp. 2, 5]. As the circumplex is built on self-reports on the meanings of emotion 
TABLE 3

Overview of the Main Theories Used in Surveyed CMEs

\begin{tabular}{lll}
\hline Theory & Abbr. & References \\
\hline Izard & Iz. & {$[108],[109],[110]$} \\
\hline Ekman & Ek. & {$[111],[112]$} \\
\hline Plutchik & Plu. & {$[113],[114]$} \\
\hline Valence \& Arousal & V-A & {$[115],[116],[117]$} \\
$\begin{array}{l}\text { Pleasure-Arousal- } \\
\text { Dominance Space }\end{array}$ & PAD & {$[118]$} \\
\hline Frijda & Frj. & {$[119],[120]$} \\
Lazarus & Laz. & {$[121]$} \\
\hline Scherer & Sch. & {$[122]$} \\
Roseman & Ros. & {$[123],[124],[125]$} \\
\hline Ortony, Clore, \& Collins & OCC & {$[126],[127],[128]$} \\
Smith \& Kirby & S \& K & {$[129],[130]$} \\
\hline Oatley \& Johnson-Laird & O \& JL & {$[131],[132],[133],[134]$} \\
Sloman & Slo. & {$[135]$} \\
\hline Damasio & Dam. & {$[136]$} \\
LeDoux & LD & {$[137]$} \\
\hline \hline
\end{tabular}

words [114, pp. 204] it is a viable candidate for CMEs concerned with analyzing text [29, pp. 218], [30, pp. 148149], though some question its validity [143, pp. 296, 298].

While Plu. proposes what the circumplex's dimensions could be [114, pp. 208], some CMEs use their own [30, pp. 153], [59, pp. 212]. The circumplex structure only constrains the relative positions of variables, and axes are arbitrary reference points [144, pp. 13]. This means that defining axes differing from Plu. is viable. Other changes have been made to Plu. based on CME needs [145, pp. 97], suggesting that Plu.'s structure is robust.

Plu. has one of the better developed theories of emotion mixing [137, pp. 113], though it is not a unique feature [141, pp. 64-65]. It uses a 3D color space analogy, which is familiar to computer scientists [22, pp. 21]. Experiments show laypeople tend to agree on the components of emotion "mixtures" [114, pp. 204-205], suggesting that Plu. is a convenient way to keep the structure of emotion categories while affording the flexibility to add more as needed [29, pp. 218], [24, pp. 35] ${ }^{2}$, [59, pp. 211, 214]. While such mixtures may not have been empirically validated, a CME's intended domain might allow their use [25, pp. 63].

Rather than basing emotion primality on facial expression-like Ekman and Izard [146, pp. 47-48], [141, pp. 83]) - Plutchik selected them based on evidence of a finite set of adaptational behaviors that aim to maintain internal homeostasis by acting on the environment [114, pp. 203, 215]. This effectively connects behaviors to action tendencies [119, pp. 72] and motivations [2, pp. 13], which could be useful for supporting neurobiological-based CMEs [55, pp. 129, 138], [22, pp. 31, 85], [24, pp. 35] $]^{3}$ and CMEs that integrate drives. There is also evidence that the circumplex can act as a common space for different types of affect [144, pp. 30-31], which can help visualize affective dynamics in combination with Plu.'s color wheel analogy [59, pp. 210-211].

2. Inferred from InFra's (17) design goals [24, pp. 27].

3. See footnote 2 .
The focus on "everyday" understandability of emotion and its structure suggests that Plu. might be an excellent choice for creating tools for integrating affect into applications and natural language processing. However, the lack of cultural specificity might lead to interpretation issues.

\subsection{Dimensional Theories}

Dimensional theories define a coordinate space using two or more affective dimensions, such as valence and arousal [4, pp. 97], [73, pp. 280]. Psychologists have located discrete emotions in this space [7, pp. 9], which is useful for understanding and debugging CMEs.

\subsubsection{Valence \& Arousal (Systems 6, 22, 41-42, 45-47)}

Valence and Arousal (V-A) appear in CMEs that typically have a limited, well-defined domain requiring real-time computations [54, pp. 766], [50, pp. 199-200], [53, pp. 23]. V-A is a simple model of affect that captures most affective phenomena, which can provide an alternate view of affective states, contribute to affective intensity evaluations [13, pp. 136], and connect a CME to other modules such as speech and body gesture generation [42, pp. 162]. V-A is also part of core affect [147, pp.170], which might be the only generalization that can be made about affective phenomena [42, pp. 151]. V-A are the two most widely agreed on affective dimensions [1, pp. 168], and are common in dimensional theories [73, pp. 280], such as the more structured circumplex models. Circumplex models could be unified with appraisal models (Section 2.3), and connected to processes such as reinforcement learning [138, pp. 279280].

Kismet (47) uses a dimensional space to combine disparate information sources, and unify the emotion elicitation process, internal representation, and facial expression generation [55, pp. 133, 148, 151]. Its authors claim that this unification helped Kismet's emotion and behavioral systems work in concert to produce the right behavior, at the right time, and in the right way to enhance its believability and understandability. However, they also found that a third dimension, stance, was needed to prevent accidental activation of emotions that are similar in the simpler $2 \mathrm{D}$ space [55, pp. 139-140]. This suggests that V-A is ideal for CMEs with a set of emotions that are conceptually easy to distinguish both as internal representations and external expressions so that additional dimensions are unnecessary.

\subsubsection{Pleasure-Arousal-Dominance Space (Systems 8, 13-14, 16, 36, 38-40, 52, 56-58, 62)}

Pleasure-Arousal-Dominance (PAD) Space appears in CMEs that want to model multiple types and functions of affectusually emotion, mood, and personality - and their interactions in a simple, unified way [22, pp. 92, 100], [49, pp. $216-$ 217, 224], [67, pp. 38], [57, pp. 217]. This aligns with the motivation to create PAD, although it mainly focuses on emotion and personality [118, pp. 261-262]. The empirical nature and ability to represent any emotion using three continuous dimensions might make PAD easy to understand with parallels to RGB color space [66, pp. 339], and especially suitable for CMEs [68, pp. 212]. The Soul (57) also connected 


\begin{tabular}{|c|c|c|c|c|c|c|c|c|c|c|c|c|c|c|c|c|}
\hline & & Iz. & Ek. & Plu. & V-A & PAD & Frj. & Laz. & Sch. & Ros. & OCC & $S \& K$ & $O \& J L$ & Slo. & Dam. & LD \\
\hline 1 & AffectR & - & - & - & - & - & - & - & - & - & $\mathrm{g}$ & - & - & - & - & - \\
\hline 2 & Cathexis & g & G & - & - & - & - & - & - & - & - & - & $\mathrm{g}$ & - & G & G \\
\hline 3 & EmMod & - & - & - & - & - & - & - & - & - & $\mathrm{g}$ & - & - & - & - & - \\
\hline 4 & FLAME & - & - & - & - & - & - & - & - & g & g & - & - & - & - & $\mathrm{g}$ \\
\hline 5 & SCREAM & - & - & - & - & - & - & - & - & - & $\mathrm{g}(60)$ & - & - & - & - & - \\
\hline 6 & MAMID & - & - & - & $\mathrm{g}$ & - & - & - & g & - & - & $\mathrm{g}$ & - & - & - & - \\
\hline 7 & TABASCO & - & - & - & - & - & $\mathrm{g}$ & - & $\mathrm{g}$ & - & - & $\mathrm{g}$ & - & - & - & - \\
\hline 8 & WASABI & - & G & - & - & G & - & - & - & - & $\mathrm{g}$ & - & - & G & - & G \\
\hline 9 & Maggie & - & - & - & - & - & - & - & - & - & $\mathrm{G}^{4}$ & - & - & - & - & - \\
\hline 10 & AKR & - & $\mathrm{g}$ & - & - & - & $\mathrm{g}$ & - & g & $\mathrm{g}$ & $\mathrm{g}$ & - & - & - & - & - \\
\hline 11 & GVH & - & G & - & - & - & - & - & - & - & $\mathrm{g}$ & - & - & - & - & - \\
\hline 12 & ParleE & - & - & - & - & - & - & - & - & G & G $(4,60)$ & - & $\mathrm{g}$ & - & - & - \\
\hline 13 & PMES & - & $\mathrm{g}^{1}$ & - & - & - & - & - & - & - & $\mathrm{g}^{1}$ & - & - & - & - & - \\
\hline 14 & IM-PMEB & - & - & - & - & - & - & - & - & - & $\mathrm{g}$ & - & - & - & - & - \\
\hline 15 & DER & G & G & - & - & - & - & - & - & - & - & - & - & - & - & - \\
\hline 16 & GenIA $^{3}$ & - & - & - & - & - & - & $\mathrm{g}(26)$ & - & - & $\begin{array}{c}\mathrm{g}(26,38, \\
43)\end{array}$ & - & - & - & - & - \\
\hline 17 & InFra & - & - & $\mathrm{g}$ & - & - & - & - & $\mathrm{g}$ & - & g (4) & - & - & - & - & - \\
\hline 18 & FAtiMA-M & - & - & - & - & - & - & - & - & - & G (35) & - & - & - & - & - \\
\hline 19 & HybridC & - & G & - & - & - & - & - & G & G & G & - & $\mathrm{g}$ & - & - & - \\
\hline 20 & SOM & - & - & $\mathrm{g}$ & - & - & - & - & - & - & - & - & - & - & - & - \\
\hline 21 & HoE & - & - & G & - & - & - & - & - & - & - & - & - & - & - & - \\
\hline 22 & Soar & - & - & - & - & - & - & - & $\mathrm{G}^{2}$ & - & - & - & - & - & - & - \\
\hline 23 & LIDA & - & - & - & - & - & - & - & $g^{2}$ & - & - & - & - & - & - & - \\
\hline 24 & CLARION & - & - & - & - & - & - & - & $\mathrm{g}$ & - & - & - & - & - & - & - \\
\hline 25 & ACRES & - & - & - & - & - & G & - & - & - & - & - & - & - & - & - \\
\hline 26 & EMA & - & - & - & - & - & - & g & - & - & $\mathrm{g}(1,29)$ & - & - & - & - & - \\
\hline 27 & Will & - & - & - & - & - & G & - & - & - & - & - & - & - & - & - \\
\hline 28 & ELSA & - & - & - & - & - & - & - & G & - & - & - & - & - & - & - \\
\hline 29 & Émile & - & - & - & - & - & - & G & - & - & $\mathrm{g}(1,60)$ & - & $\mathrm{g}$ & - & - & - \\
\hline 30 & EMOTION & - & - & - & - & - & - & - & - & - & $\operatorname{g~}(11)^{6}$ & - & - & - & - & - \\
\hline 31 & JBdiEmo & - & - & - & - & - & - & - & - & - & $\mathrm{g}^{3}$ & - & - & - & - & - \\
\hline 32 & DETT & - & - & - & - & - & - & - & - & - & G & - & - & - & - & - \\
\hline 33 & Puppet & - & G & - & - & - & - & - & - & - & G & - & - & - & - & - \\
\hline 34 & CBI & - & - & - & - & - & - & G & - & - & - & - & - & - & - & - \\
\hline 35 & FAtiMA & - & - & - & - & - & - & $\mathrm{g}(26)$ & - & - & $\mathrm{g}(26)$ & - & - & - & - & - \\
\hline 36 & TARDIS & - & - & - & - & - & - & - & - & - & $\mathrm{g}$ & - & - & - & - & - \\
\hline 37 & Greta & - & - & - & - & - & - & - & - & - & G & - & - & - & - & - \\
\hline 38 & ALMA & - & - & - & - & - & - & - & - & - & $\mathrm{g}$ & - & - & - & - & - \\
\hline 39 & Eva & - & - & - & - & - & - & - & - & - & $\mathrm{g}^{4}$ & - & - & - & - & - \\
\hline 40 & PPAD-Algo & - & - & - & - & G & - & - & - & - & g (38) & - & - & - & - & - \\
\hline 41 & PSA Heart & - & - & - & G & - & - & - & - & - & - & - & - & - & - & - \\
\hline 42 & Peedy & - & - & - & G & - & - & - & - & - & - & - & - & - & - & - \\
\hline 43 & ERDAMS & - & - & - & - & - & - & - & - & - & G $(1,60)$ & - & - & - & - & - \\
\hline 44 & TEATIME & - & - & - & - & - & G & - & - & $\mathrm{g}$ & - & - & - & - & - & - \\
\hline 45 & Presence & - & - & - & - & - & - & - & - & - & G & - & - & - & - & - \\
\hline 46 & FFSM-Emo & - & - & $\mathrm{g}$ & G & - & - & - & - & - & - & - & - & - & - & - \\
\hline 47 & Kismet & G & - & G & $\mathrm{G}^{5}$ & - & - & - & - & - & - & - & - & - & G & G \\
\hline 48 & KaMERo & - & - & - & - & - & - & - & - & - & G & - & - & - & - & - \\
\hline 49 & R-Cept & - & $\mathrm{g}$ & - & - & - & - & - & - & - & - & - & - & - & - & - \\
\hline 50 & POMDP-CA & - & - & - & - & - & - & - & - & G & - & - & - & - & - & - \\
\hline 51 & TAME & - & G & - & - & - & - & - & - & - & - & - & - & - & - & - \\
\hline 52 & iPhonoid & - & G & - & - & $\mathrm{g}$ & - & - & - & - & G & - & - & - & - & - \\
\hline
\end{tabular}


TABLE 4

(Continued.) Theories Used for Emotion Generation in CMEs

\begin{tabular}{|c|c|c|c|c|c|c|c|c|c|c|c|c|c|c|c|c|}
\hline & & Iz. & Ek. & Plu. & V-A & PAD & Frj. & Laz. & Sch. & Ros. & OCC & $S \& K$ & O \& JL & Slo. & Dam. & LD \\
\hline 54 & GRACE & - & - & - & - & - & - & - & $\mathrm{g}$ & - & $\mathrm{g}$ & - & - & - & - & - \\
\hline 56 & SocioEmo & - & - & - & - & - & - & - & - & - & $\mathrm{G}^{4}$ & - & - & - & - & - \\
\hline 57 & The Soul & - & - & - & - & G & - & - & - & - & $\mathrm{g}(38)$ & - & - & - & - & - \\
\hline 60 & $\mathrm{Em} / \mathrm{Oz}$ & - & - & - & - & - & - & - & - & - & G & - & - & - & - & - \\
\hline 61 & S3A & - & - & - & - & - & $\mathrm{g}(27)$ & - & - & - & G & - & - & - & - & - \\
\hline 62 & FeelMe & - & - & - & - & G & - & - & G & - & - & G & - & - & - & - \\
\hline 63 & INVITE-A & - & - & - & - & - & - & - & - & - & $\mathrm{G}^{3}$ & - & G & - & - & - \\
\hline
\end{tabular}

G: Reasons for choosing the theory are clear; g: Reasons are unclear; (\#): System borrowed from/is influenced by System \#

${ }^{1}$ It is unclear if it uses this theory [21, pp. 253].

${ }^{2}$ Not yet implemented [138, pp. 271], [32, pp. 26].

${ }^{3}$ Uses the OCCr model [40, pp. 195, 197], [72, pp. 17] a reinterpretation of the OCC model that aims to clarify the model's logical structure and address ambiguities [139, pp. 1].

${ }^{4}$ Based on [127, pp. 193], a simplified model developed by Ortony for believable "artifacts" [48, pp. 23], [12, pp. 62], [65, pp. 285].

${ }^{5}$ Also uses a stance dimension to measure the approachability of a stimulus [55, pp. 133].

${ }^{6}$ Builds on [86, pp. 2], a successor of GVH (11).

TABLE 5

Theories Used for Emotion Expression in Surveyed CMEs

\begin{tabular}{|c|c|c|c|c|c|c|c|c|c|c|c|}
\hline & & Iz. & Ek. & Plu. & V-A & PAD & Frj. & Laz. & OCC & Slo. & Dam. \\
\hline 1 & AffectR & - & - & - & - & - & - & - & $x^{1}$ & - & - \\
\hline 2 & Cathexis & - & - & - & - & - & - & - & - & - & $X$ \\
\hline 3 & EmMod & - & $X$ & - & - & - & - & - & - & - & - \\
\hline 5 & SCREAM & - & $X$ & - & - & - & - & - & $x^{2}$ & - & - \\
\hline 7 & TABASCO & - & - & - & - & - & $X$ & $x$ & - & - & - \\
\hline 8 & WASABI & - & $X$ & - & - & - & - & - & - & - & - \\
\hline 11 & GVH & - & $X$ & - & - & - & - & - & - & - & - \\
\hline 12 & ParleE & $X$ & $X$ & - & - & - & - & - & - & - & - \\
\hline 16 & GenIA $^{3}$ & - & - & - & - & - & - & $x(26)$ & $x^{2}$ & - & - \\
\hline 24 & CLARION & - & - & - & - & - & - & $x$ & - & - & - \\
\hline 25 & ACRES & - & - & - & - & - & $\bar{X}$ & - & - & - & - \\
\hline 26 & EMA & - & - & - & - & - & - & $x$ & - & - & - \\
\hline 29 & Émile & - & - & - & - & - & - & - & - & - & $x$ \\
\hline 33 & Puppet & - & $X$ & - & - & - & - & - & - & - & - \\
\hline 34 & CBI & - & - & - & - & - & - & $X$ & - & - & - \\
\hline 35 & FAtiMA & - & - & - & - & - & - & $X(26,34)$ & - & $X$ & - \\
\hline 37 & Greta & - & $X$ & - & - & - & - & - & - & - & - \\
\hline 45 & Presence & - & - & - & $X$ & - & $x$ & - & - & - & - \\
\hline$\overline{47}$ & Kismet & $X$ & $x$ & $X$ & $\mathbf{X}^{3}$ & - & - & - & - & - & - \\
\hline 54 & GRACE & - & - & - & - & - & - & $x$ & - & - & - \\
\hline 57 & The Soul & - & $X$ & - & - & $\mathrm{X}$ & - & - & - & - & - \\
\hline 60 & $\mathrm{Em} / \mathrm{Oz}$ & - & - & - & - & - & - & - & $\mathbf{X}^{1}$ & - & - \\
\hline 61 & S3A & - & - & - & - & - & - & - & $X(60)$ & - & - \\
\hline
\end{tabular}

$\mathrm{X}$ : Reasons for choosing the theory are clear; $\mathrm{x}$ : Reasons are unclear; (\#): System borrowed from/is influenced by System \#

${ }^{1}$ Based on an unpublished work which AffectR describes [10, pp. 50] and Em/Oz duplicates and expands on [70, pp. 104].

2 Based on [127, pp. 193, 198], a simplified model developed by Ortony for believable "artifacts" [14, pp. 234], [27, pp. 5:5].

3 Also uses a stance dimension to measure the approachability of a stimulus [55, pp. 133, 140]. 
TABLE 6

Theories Used for Other Purposes in Surveyed CMEs

\begin{tabular}{|c|c|c|c|c|c|c|c|c|c|c|c|c|c|c|c|}
\hline & & Iz. & Plu. & V-A & PAD & Frj. & Laz. & Sch. & Ros. & OCC & $S \& K$ & $\mathrm{O} \& \mathrm{JL}$ & Slo. & Dam. & LD \\
\hline 3 & EmMod & - & - & - & - & - & - & - & - & - & - & - & - & $\mathrm{O}$ & - \\
\hline 5 & SCREAM & - & - & - & - & - & - & - & - & $\mathrm{o}^{1}$ & - & - & - & - & - \\
\hline 7 & TABASCO & - & - & - & - & O & - & - & - & - & - & - & - & - & - \\
\hline 8 & WASABI & - & O & - & - & - & - & o & - & O & - & - & O & O & o \\
\hline 9 & Maggie & - & - & - & - & - & O & - & - & - & - & - & - & - & - \\
\hline 13 & PMES & - & - & - & o (38) & - & - & - & - & - & - & - & - & - & - \\
\hline 14 & IM-PMEB & - & - & - & o (38) & - & - & - & - & - & - & - & - & - & - \\
\hline 15 & DER & - & - & - & - & - & - & - & - & - & - & - & o & o & - \\
\hline 16 & GenIA $^{3}$ & - & - & - & o (38) & - & - & - & - & - & - & - & - & - & - \\
\hline 17 & InFra & - & - & - & - & - & - & - & - & - & - & - & - & - & o \\
\hline 18 & FAtiMA-M & - & - & - & - & - & O & O & - & - & - & - & - & - & - \\
\hline 19 & HybridC & $\mathbf{O}$ & $\mathbf{O}$ & - & - & - & - & - & - & - & - & - & - & - & - \\
\hline 22 & Soar & - & - & O & - & - & - & - & - & - & - & - & - & - & - \\
\hline 24 & CLARION & - & - & - & - & - & - & - & - & $\mathbf{O}$ & - & - & - & - & - \\
\hline 26 & EMA & - & - & - & - & o & - & o & - & - & o & - & - & - & - \\
\hline 29 & Émile & - & - & - & - & - & - & - & - & - & - & - & $\mathrm{O}$ & - & - \\
\hline 35 & FAtiMA & - & - & - & - & o (61) & - & - & - & o & - & - & - & - & - \\
\hline 36 & TARDIS & - & - & - & o $(38,56)$ & - & - & - & - & - & - & - & - & - & - \\
\hline 37 & Greta & - & - & - & - & - & - & - & - & - & - & o & - & - & - \\
\hline 38 & ALMA & - & - & - & o & - & - & - & - & - & - & - & - & - & - \\
\hline 39 & Eva & - & - & - & o (38) & - & - & - & - & - & - & - & - & - & - \\
\hline 40 & PPAD-Algo & - & - & - & o $(38,39)$ & - & - & - & - & - & - & - & - & - & - \\
\hline 41 & PSA Heart & - & - & - & - & - & - & - & - & o & - & - & - & - & - \\
\hline 43 & ERDAMS & - & - & - & - & - & - & $\mathbf{O}$ & $\mathbf{O}$ & $\mathbf{O}$ & - & - & - & - & - \\
\hline 45 & Presence & - & - & - & - & - & - & - & - & - & - & - & o & $\mathrm{o}$ & - \\
\hline 48 & KaMERo & - & - & - & - & - & - & - & - & o & - & - & o & - & - \\
\hline 49 & R-Cept & - & - & - & - & - & - & o & - & - & - & - & - & - & - \\
\hline 51 & TAME & - & - & - & - & $\mathbf{O}$ & - & - & - & - & - & - & - & - & - \\
\hline 53 & PWE-I & - & - & - & - & - & - & o & - & - & - & - & - & - & - \\
\hline 55 & EEGS & - & - & - & - & - & - & - & - & $\mathbf{O}$ & - & - & - & - & - \\
\hline 56 & SocioEmo & - & - & - & $\mathbf{O}(38)$ & - & - & - & - & - & - & - & - & - & - \\
\hline 57 & The Soul & - & - & - & o (38) & - & - & - & - & - & - & - & - & - & - \\
\hline 58 & GAMYGDALA & - & - & - & O & - & - & - & - & - & - & - & - & - & - \\
\hline 59 & MM & - & - & - & - & o (27) & - & - & - & - & - & o & - & - & - \\
\hline 61 & S3A & - & - & - & - & $\mathbf{O}(27)$ & - & - & - & - & - & - & - & - & - \\
\hline
\end{tabular}

O: Reasons for choosing the theory are clear; o: Reasons are unclear; (\#): System borrowed from/is influenced by System \#

${ }^{1}$ Based on [127, pp. 193], a simplified model developed by Ortony for believable "artifacts" [14, pp. 231].

facial expression generation to PAD Space [66, pp. 340-342], creating a unified emotion generation/expression CME.

PAD is very similar to V-A. Its pleasure dimension fills the same role as valence, and arousal is shared by both theories. The third dimension, dominance, distinguishes between emotions such as Anger and Fear [118, pp. 264]. WASABI (8) found this necessary in its design, but stated that dominance cannot be evaluated without cognition [22, pp. 58, 100]. Thus PAD should be used with deliberative mechanisms, although ALMA (38) and The Soul (57) do not have any. However, their goals are not geared toward affective research.

PPAD-Algo (40) defines emotion intensity as the length of a vector in PAD space [49, pp. 217], based on ideas for a two-dimensional affective space [148]. While it is unclear if the dimensions are homogeneous, the results appear "good enough" for non-research applications. Emotion intensity measures have not historically received as much agreement compared to other facets of affective science [149], [6, pp. 373] and many of the theories examined here also do not address intensity comprehensively.

PAD also tends to appear in CMEs that model mood, using a set of fixed values to represent personality as the initial mood point. ALMA (38) might have been first to do so [47, pp. 31]. However, this use might be due to the translation error from English to German [150, pp. 354] as PAD describes a personality space-not mood [118, pp. 266267]. Still, many CMEs have adopted ALMA's mood model, suggesting it is "good enough" [65, pp. 289] for many applications. Indeed, ALMA and the CMEs that borrow its mood model [23, pp. 68], [27, pp. 5:18], [48, pp. 24], [49, pp. 217, 222], [66, pp. 339], [21, pp. 255], [45, pp. 5] 
are mainly concerned with believability. The exception is WASABI (8), intended for both believability and affective research [22, pp. 10], and does not reference ALMA. ALMA also connects PAD to OCEAN personality traits [151] via [118]'s mapping [47, pp. 31]. ALMA's predecessors might have motivated this choice [90, pp. 52], [91, pp. 131]. ALMA's PAD-based model of personality better aligns with the theory's intent, and has been reused in [65, pp. 289] and [45, pp. 5], as well as those that use its mood model. Overall, ALMA is a good PAD starting point for CMEs emphasizing believability over validity. For a very simple mood model, coherent with Ek.'s concept of mood, the pleasure dimension can act as an approximation of good and bad mood [57, pp. 218].

PAD's pervasiveness in CMEs suggests its usefulness for creating a unified space for multiple types of affect and interfacing between theories. Caution is required as soundness depends on how rigorously concepts are matched.

\subsection{Appraisal Theories}

Appraisal theories emphasize distinct components of emotion, including appraisal dimensions or variables [4, pp. 97], and the role of cognition in emotion elicitation [6, pp. 354]. They suggest that subjective appraisals of events or potentially uncontrolled, unconscious cognitive evaluations elicit most emotions [73, pp. 280]. Appraisal variables map onto an $n$ dimensional space, where specific configurations represent specific emotions [140, pp. 306]. Individuals generate values for these variables by analyzing stimuli for personal meaning and consequences, and often their goals [152, pp. 250].

\subsubsection{Frijda (Systems 7, 10, 25-27, 35, 44-45, 51, 59, 61)}

Frijda (Frj.) often appears in CMEs to explicitly connect emotions to action tendencies and define an action-driven appraisal process [34, pp. 247], [36, pp. 151], [52, pp. 146, 150]. This aligns with Frj.'s proposal that emotions-outputs of a continuous information processing system-are changes in action readiness [119, pp. 453, 466]. "Action readiness" refers to motivational states, which are associated with goals rather than actions or behaviors [120, pp. 143]. For CMEs, this affords the freedom to design flexible, domainindependent goal-achievement processes that multiple affective states can use. CMEs can thus use action evaluations to drive affective processes [17, pp. 268-269]. For systems like TEATIME (44), whose focus is a flexible affective decision-making mechanism [52, pp. 143], this connection is essential. Frj.'s description of action tendencies appears to transfer to designs that do not implement its appraisal process [17, pp. 267], [42, pp. 161], [18, pp. 594, 596-597], since many of the identified action tendencies are associated with an emotion label [119, pp. 87-90].

The conceptualization of emotion elicitation as an information processing system is a useful analogy for CMEs, and can provide the necessary mechanization framework for structure-oriented theories like Ros. [52, pp. 146] and OCC [71, pp. 37, 48, 61]. It is also possible to abstract and apply different elements independently of the broader theory, such as implicit appraisal checks [17, pp. 266], information filtering [71, pp. 49], and mechanisms whose behavior changes with the system state [63, pp. 211], [37, pp. 286, 297]. The Will architecture (27), built on Frijda's definition, relates emotion to personality, mood, and sentiments, allowing it to define them as system configurations [36, pp. 135-136, 157-158]. These ideas have been adopted by other CMEs, requiring fewer subsystems in their implementation [69, pp. 6-7, 9], [71, pp. 48], [44, pp. 130-132].

\subsubsection{Lazarus (Systems 7, 9, 16, 18, 24, 26, 29, 34-35, 54)}

Lazarus (Laz.) usually appears when a CME requires coping behavior [43, pp. 302, 306], [33, pp. 10, 12], [27, pp. 5:5-5:6], a deliberative process whereby the individual can suppress action tendencies and choose other strategies for influencing the current situation [153, pp. 628]. As coping plays a critical role in the theory [121, pp. 39-40], the appearance of Laz. in this context is unsurprising. Coping has been incorporated directly into the appraisal process as an influencing factor [37, pp. 272, 279]. It has also been used alone for planning agent behaviors [17, pp. 267], [37, pp. 278], [62, pp. 135], [38, pp. 325, 327, 331], and has been successfully paired with a separately defined component for quick, reactionary behaviors [44, pp. 130, 132-133]. The coping models of EMA (26), Émile (29), and CBI (34) have been particularly influential [154, pp. 353], [155].

Laz. also describes a reappraisal process to explain the continuous and responsive nature of the emotion system to changes in the environment [121, pp. 134]. This is directly tied to coping, which can affect changes in an individual's interpretation of the environment. This concept has also appeared alone, in CMEs that reprocess information after deliberative processes like coping [44, pp. 133-134], [28, pp. 46-47], which could result in different emotions compared to purely reactive systems.

Another feature of Laz. is its use of relational themes, which are connected to emotions [121, pp. 122]. This treats appraisal as a comprehensive unit rather than a set of individual dimensions. This emulates discrete categories, allowing CMEs to treat each emotion separately [12, pp. 60].

2.3.3 Scherer (Systems 6-8, 10, 17-19, 22-24, 26, 28, 43, $49,53-55,62)$

Scherer (Sch.) tends to appear where CME designs need multi-level and/or multi-stage appraisals [68, pp. 210-211], [13, pp. 136] that incorporate cognition in their evaluations $[25, \mathrm{pp} .61,66]$. These features are inherent in Sch. [122, pp. 99, 103]. This group of CMEs notably include the Cognitive Architectures [138, pp. 272, 274], [32, pp. 26-27], [33, pp. 9, 11], which is likely a result of Sch.'s complexity compared to other appraisal theories [28, pp. 46-47], [156, pp. 58]. It uses a dynamic systems view where components change and interact over time, such that emotion is an emergent product of system synchronization [35, pp. 99$102,143]$. Sch. has used neural networks as a system representation.

However, other CMEs have found ways to use Sch. without using multi-level or multi-stage processes. Some CMEs have opted to use only its appraisal variables [17, pp. 266], [18, pp. 597], sometimes combining them with variables from other theories like OCC [24, pp. 30], [157, pp. 8-9], and emotion descriptions because they account for coping potential [51, pp. 416-417]. Others take inspiration from 
its process model to connect emotion generation to other subsystems [62, pp. 138]. Still others reference Sch. for some of its theoretical concepts [158, pp. 141-142], [122, pp. 9596], such as the design-oriented differentiation between types of affect [22, pp. 83], [61, pp. 323], [59, pp. 209], and to show how goals can represent a broad range of motivational states [37, pp. 286, 297]. This suggests that some CMEs can comfortably use pieces of Sch. independently of the complete theory.

\subsubsection{Roseman (Systems 4, 10, 12, 19, 43-44, 50)}

Roseman (Ros.) appears in CMEs to map emotions to action tendencies, and to define Surprise as an emotion. Ros. is usually not used alone. In TEATIME (44), Ros. is combined with Frj. because they both emphasize action tendencies which are essential to its target application [52, pp. 143, 146]. POMDP-CA (50) is an exception, using Ros. to represent cognitive appraisal theories [56, pp. 268]. This might be because, out of the two theories identified in [1] for cognitive appraisal, Ros. systematically built a model between appraisal variables and emotions from empirical studies [123, pp. 267-268] which makes it more plausible [56, pp. 265].

The larger issue is that Ros. does not specify an emotion generation process. CMEs have compensated for this by using Markov Models [56, pp. 267], fuzzy logic [11, pp. 227228], and combining Ros. with process-based theories like Sch. [25, pp. 66] and Frj. [52, pp. 150].

CMEs that use Ros. to define Surprise use other theories-usually Sch. and/or OCC - that do not explicitly define it [18, pp. 597], [20, pp. 119, 135-136], [25, pp. 66]. These unions appear to be sound. OCC agrees with Ros. that unexpectedness elicits Surprise [126, pp. 32], and Sch.'s suddenness variable in the novelty check appears to do a comparable evaluation [122, pp. 95]. Anger is an emotion that Ros. shares with OCC, but it limits its scope to events caused by other agents, which a CME might find more helpful [51, pp. 417].

2.3.5 Ortony, Clore, \& Collins (Systems 1, 3-5, 8-14, 16$19,24,26,29-33,35-41,43,45,48,52,54-58,60-61,63)$

The Ortony, Clore, and Collins (OCC) model of emotion is the most used [72, pp. 16], [80, pp. 292], [62, pp. 136] and widely accepted theory in affective computing [39, pp. 1] despite cautioning that "...we view each emotion specification, or characterization, as a proposal rather than as an empirically established fact." [126, pp. 87-88], and not being as popular in psychology [37, pp. 278]. The widespread use of OCC is partially due to its hierarchical structure of emotions and eliciting conditions, which feels familiar to computer scientists [22, pp. 44], [156, pp. 57] and is amenable to computation [126, pp. 2, 181-182], [1, pp. 195], [7, pp. 8], [6, pp. 362]. It also accounts for some emotions about others, but does not provide a full description of emotion intensity or the process of emotion elicitation.

2.3.5.1 Computational Tractability: Some CMEs mention, directly or indirectly, that its prevalence in affective computing is a reason for choosing OCC [25, pp. 61], [67, pp. 33], [72, pp. 16-17], [21, pp. 251, 253] $]^{4}$. Others have compared it to a computational approach [70, pp. 28], [25,

4. This decision is inferred. pp. 66], [62, pp. 136], [67, pp. 33] or its relative ease of implementation [65, pp. 282], [20, pp. 135-136], [71, pp. 48], [44, pp. 129-130]. Still, these reasons alone should be insufficient for choosing OCC over other theories-it is not a "silver bullet" solution [159, pp. 2]. Computer scientists pushed OCC's computability by resolving ambiguities and creating an inheritance-based structure, but they caution that its integrity as a psychological theory might have been compromised [139, pp. 1]. However, this is not a concern for CMEs that are not meant to simulate actual affective phenomena [40, pp. 195], [72, pp. 9, 17]. A further benefit of OCC's comparison to a computational approach is that it can be easier to understand without a background in psychology [70, pp. 28], [65, pp. 282]. This might make it more "clear and convincing" [97, pp. 741] than other appraisal theories.

OCC assumes that people view the world in terms of world aspects and changes [126, pp. 18]. One could view this as an event-driven model, which also lends itself to computational modeling [54, pp. 766], [94, pp. 264], [46, pp. 94], [62, pp. 138], [71, pp. 51], [21, pp. 253], [45, pp. 4], [58, pp. 236], [42, pp. 151-152]. This view led some CMEs to define what emotions they can produce as they relate to eliciting events [14, pp. 230], [39, pp. 4], [44, pp. 129], [48, pp. 23], [51, pp. 412,418$],[67$, pp. 33], [157, pp. 4, 8-9], [25, pp. 66], [23, pp. 68], which can be independent of cognitive processing [19, pp. 109].

OCC also describes how to structure different types of goals, attitudes, and standards, relating them to the emotion hierarchy and each other [126, pp. 34-35]. This has motivated OCC's adoption as they make it clearer how emotions and behaviors are related to both goal [33, pp. 10] and nongoal structures [20, pp. 136], [62, pp. 138], [157, pp. 6], how to differentiate between goal types [44, pp. 131], and how to relate goals in the hierarchy [71, pp. 51], [24, pp. 31].

Ironically, OCC's authors believe that computers cannot have emotion but it is still useful to reason about them: "...we do not consider it possible for computers to experience anything until and unless they are conscious. Our suspicion is that machines are simply not the kinds of things that can be conscious...There are many AI [Artificial Intelligence] endeavors in which the ability to understand and reason about emotions or aspects of emotions could be important" [126, pp. 182]. AffectR (1) adheres to this when reasoning about another agent's actions [10, pp. 27]. However, OCC can be applied to emotion generation as well [1, pp. 195], also shown by AffectR, because the process of reasoning about emotions could be understood as reasoning about the emotional significance of an event to the agent [14, pp. 230]. The focus on reasoning makes OCC amenable to an intentional stance, which enhances agent believability [42, pp. 151-152], because users can "see" the agent's thought processes. Ortony later created a version of OCC specifically for affective agents, focusing on consistent and believable behaviors [127]. This is ideal for CMEs that do not need to simulate true affective phenomena, but only a few use it [65, pp. 285], [12, pp. 62], [48, pp. 21]. Eva (39), one of the systems using [127], has been extended [160, pp. 2], hinting at the effectiveness of [127] for affective agents.

Unlike the original OCC theory, [127] includes a hierarchy of response tendencies grouped by type which CMEs 
have used [14, pp. 234], [27, pp. 5:5]. This might be a simplification of unpublished work intended for the full theory, which AffectR (1)—a research model—and Em/Oz (60) - a non-research model-incorporate [10, pp. 51], [70, pp. 28, 104]. This hierarchy can be implemented such that it allows users to categorize display mechanisms [10, pp. 5153], [14, pp. 234] —encouraging modular development-and assign the same behavior to different tendencies, affording more control over emotional displays [70, pp. 86, 100], [71, pp. 53].

2.3.5.2 Empathetic Emotions and Surprise: Something that might be unique to OCC are the "fortunes of others" emotions (e.g. Happy-For), which rely on evaluations of how someone else feels. These are critical for empathetic agents [51, pp. 412], [46, pp. 97, 111] and agents that model relationships [65, pp. 288], [45, pp. 1, 4], [67, pp. 33], [72, pp. 33, 48]. Eva (39) uses OCC to connect to a non-affective relationship model so that it can establish relationships with users [48, pp. 22-23]. However, OCC does not include Surprise which is important for some CMEs. OCC excludes Surprise because they believe that it is not inherently positive or negative [126, pp. 32]. Instead, they categorize it as a cognitive state tied to a global unexpectedness variable. CMEs that need Surprise draw from Ros. [20, pp. 119], [25, pp. 66] because it shares this hypothesis [123, pp. 269].

2.3.5.3 Computing Emotion Intensities: OCC's structure also shows which variables contribute to an emotion's intensity, proposing that it is evaluated with a weighted function [126, pp. 69, 82]. Unfortunately, it does not propose what those weights should be, nor the function's nature. AffectR (1) appears to circumvent this issue by not computing a numerical intensity value at all [10, pp. 4750]. ALMA (38) requires a separate tool to define intensity functions parameters [161, pp. 206-207], which The Soul (57) found useful for its purposes [66, pp. 339]. Other CMEs solved this by translating OCC emotion families to other theories [49, pp. 217], creating their own functions from the given variables [11, pp. 233], [51, pp. 419-422], [12, pp. 62], [67, pp. 37-38], [72, pp. 48], [157, pp. 14] or manually coding them [71, pp. 62], [44, pp. 133], [41, pp. 994-995], [22, pp. 95], [48, pp. 23], [57, pp. 220]. Em/Oz (60) opted to only adopt a subset of variables, finding that the simpler model was sufficient for its goals [70, pp. 53]. Emile (29) drew from Em/Oz specifically for its simpler intensity functions [38, pp. 328], though it is not the only CME to use Em/Oz this way [14, pp. 230]. Some CMEs have found various combinations of Em/Oz, Emile, and FLAME (4) intensity functions useful their own purposes [65, pp. 289], [20, pp. 117-125].

A model of mood might be needed to prevent unnaturally fast fluctuations in emotion state [22, pp. 88], and many CMEs that use OCC also model mood [70, pp. 75, 94], [23, pp. 68], [27, pp. 5:18], [48, pp. 24], [49, pp. 217, 222], [66, pp. 339], [21, pp. 255], [45, pp. 5], [14, pp. 231]. ALMA (38) explicitly notes that the inclusion of mood produced a more natural emotion intensity [150, pp. 354].

Strictly speaking, the weighted function used by these systems is not an intensity function. OCC proposes that a weighted combination of the EECs leading to an emotion family is an emotion potential - a higher potential means a higher chance of experiencing that emotion [126, pp. 8182]. The difference between an emotion threshold and this value is its intensity. CMEs can modulate these thresholds to implicitly model moods and personality [16, pp. 65-66], [71, pp. 51], [46, pp. 103, 109-110], [20, pp. 119], [44, pp. 131], [22, pp. 92-93], [28, pp. 48].

2.3.5.4 The Missing Emotion Elicitation Process: A shortcoming of OCC is a lack of emotion generation processes [25, pp. 65]. This is a deliberate omission, as OCC it views as a general cognitive psychology problem, although it stresses the role of cognition in such processes [126, pp. 2]. Some CMEs refer to this in their analysis of OCC [25, pp. 61], [18, pp. 597], [42, pp. 161], [23, pp. 67]. AffectR (1) might be the most complete realization of the missing processes [10, pp. 30-33]. Subsequent CMEs used AffectR's process, generalized for plans to achieve domain independence [38, pp. 326-327], [20, pp. 118] and partially simplified [37, pp. 282-283, 285], [44, pp. 130, 132]. FAtiMA's (35) implementation proved effective, as [80] adopted it specifically for its planning and appraisal mechanisms. Plans are also a step towards explainable behaviors by providing context for elicited emotions [38, pp. 328], which aligns with the OCC's focus on reasoning about emotions [126, pp. 182].

Some CMEs take a biologically-inspired approach based on motivations and drives [12, pp. 56], [23, pp. 65-66]. The reliance on cognition guided yet other CMEs to use OCC to define a deliberative layer in biologically-inspired architectures [16, pp. 63], [22, pp. 58, 100], [60, pp. 258259]. This is supported by [128, pp. 177], which incorporates neurobiological findings. Other CMEs integrate OCC into a Belief-Desire-Intention (BDI)-inspired system or architecture to account for cognitive activities [41, pp. 993], [51, pp. 417], [42, pp. 153-154, 161], [40, pp. 195], [27, pp. 5:4-5:5]. However, this can make the CME itself difficult to modify if it is integrated too deeply into the host architecture [46, pp. 111112]. Some CMEs aim to be architecture agnostic, but still require at least one BDI-like input to produce emotions [67, pp. 32, 36-37], [157, pp. 7], [14, pp. 227, 230, 234-235], [24, pp. 28-29]. The reliance on cognition has also led some CMEs to have mechanisms allowing other reasoning processes to influence emotion generation [162, pp. 213].

CMEs have also combined the OCC structures with a process-oriented theories like Frj. [71, pp. 48] and Sch. [62, pp. 138], [25, pp. 66], or draw from Picard [1] for guidance [18, pp. 603], [21, pp. 252]. Many CMEs set their emotion model between input and output modules to mediate their interactions [39, pp. 4], [90, pp. 50], [48, pp. 22], [65, pp. 284], [66, pp. 342-343], [28, pp. 45], [72, pp. 33], [49, pp. 211-212], [57, pp. 218], [19, pp. 110], with some using or considering fuzzy logic to aid in matching eliciting conditions to emotions [11, pp. 227-228], [45, pp. 3, 7], [24, pp. 2930]. If the goal is not to create "correct" behaviors, this strategy is sufficient if it meets the CME design goals [70, pp. 44-45].

\subsubsection{Smith \& Kirby (Systems 6-7, 55, 62)}

Smith \& Kirby (S \& K) only seems to appear in CMEs that want to integrate multiple, parallel input sources into one unit for appraisal. This appears to be its distinguishing factor among appraisal theories [130, pp. 129-130].

$$
\text { 5. Builds on [9, pp. 31]. }
$$


S \& K always appears with Sch. to combine appraisal information from sources from multiple levels of resolution [13, pp. 136], [17, pp. 266, 268], [68, pp. 210]. This might be because Sch. is better validated [130, pp. 129], computationally tractable, and because Scherer draws parallels between sequential check registers and S \& K's integrated appraisal [122, pp. 105, 120]. Another possibility for this pairing is a misconception that Sch. is strictly a sequential appraisal process [58, pp. 236] when it is not [122, pp. 100, 103]. One exception is EMA (26), which combines S \& K with Frj. to define an attention mechanism [37, pp. 286]. This is likely because [120, pp. 149] compares its "blackboard control structure" to $S$ \& K's appraisal register. This suggests that CMEs can combine $S \& \mathrm{~K}$ with other theories that have some comparable work to the appraisal register concept.

\subsubsection{Oatley \& Johnson-Laird ${ }^{6}$ (Systems 2, 12, 19, 29, 37, $59,63)$}

Oatley \& Johnson-Laird (O \& JL) appears in CMEs to define what emotions they support, relate other types of affect to emotions, and connect emotion intensity to changes in computational plans. O \& JL typically have a supporting role for defining emotions in CMEs. Ek. is usually the main theory present for defining CME emotions [15, pp. 71], [25, pp. 6364]. This connection is sound, as O \& JL considered Ek. as evidence when identifying their set of basic emotions [133, pp. 57-61].

Moods and personality are conceptualized in O \& JL's theory in relation to emotions [133, pp. 23-24, 61]. This is useful for CMEs because it allows them to use one representation that can be configured to for different types of affect. MM (59) references this definition of mood, but combines it with the extension of Frj. proposed by the Will architecture (27) [69, pp. 9]. It does not reference O \& JL for personality. Sentiments-long-term emotional dispositions mainly towards others-were added to the theory later [134, pp. 81], and is a natural extension to a theory emphasizing the communicative role of emotion in social structure and relationships [131, pp. 41]. For agents whose behaviors should be influenced by their relationships with others, this concept is invaluable [72, pp. 19-20].

$\mathrm{O} \& \mathrm{JL}$ propose that there is no emotion process, arguing that emotions are states entered at plan junctures, which that might include conflicts between different goals, agents, and resources demands [133, pp. 22, 24-25, 31-36]. CMEs have taken this information to define emotion intensity in relation to an agent's goals and plans [20, pp. 117], [38, pp. 328]. This also frames cognition as a knowledge transformation process, which is amenable to computation [46, pp. 94].

Perhaps the most useful element of $\mathrm{O} \& \mathrm{JL}$ is its focus on the social and communicative role of emotions [131, pp. 4142]. This has implications for multi-agent applications with affective content because each agent is an independent module in a larger system [133, pp. 178, 181-182]. Conversational agents might also benefit from this view, which casts conversations as a form of mutual planning. As the field of

6. Although it does not name appraisal dimensions, Oatley \& Johnson-Laird talk about evaluating events relevant to plans and goals individually such that changes in achievement probability induce emotions [133, pp. 50]. Therefore, it is grouped with the appraisal theories. social affective agents progresses, $\mathrm{O} \& \mathrm{JL}$ could come to play a larger role in their development.

\subsection{Neurobiological Theories}

Biological neural circuitry and brain structures inspire the neurobiological theories of affect [4, pp. 98]. Though uncommon in affective computing [163, pp. 451], they have the potential to influence biologically-inspired CMEs for applications such as embodied agents and those that have drives, limited resources, and must select actions in real time that are consistent with the agent's internal state [6, pp. 364].

\subsubsection{Sloman ${ }^{7}$ (Systems 2, 8, 15, 29, 35, 45, 48)}

Sloman (Slo.) is commonly used to define the architecture surrounding emotion generation [60, pp. 257-258], [26, pp. 3-6], [42, pp. 160], [22, pp. 84] rather than doing it directly. This is likely because Slo. conceptualizes emotion as a product of a central information-processing system [135, pp. 204, 211]. This affords the distinction between the processing requirements of emotion at different processing resolutions while considering the system's constraints. The division of processes into layers also makes it possible to adopt some of Slo.'s mechanisms independently of the broader theory. For example, Slo.'s deliberative layer contains the ability to plan [135, pp. 223]. Therefore, Slo. can help with tasks in plan-based CME components, such as evaluating goal importance based on plans [38, pp. 329], and affect-driven resource allocation based on urgency [44, pp. 134].

WASABI (8) is the only CME surveyed that uses Slo. directly in emotion generation for non-cognitive emotion elicitation [22, pp. 90], using its definition of emotions as information processing interrupts and modulators [135, pp. 211, 230]. This might be due to Slo.'s inclusion of "beliefs", "desires", and "intentions" in its architectural specification [135, pp. 208], which neatly aligns with WASABI's underlying BDI architecture.

\subsubsection{Damasio (Systems 2-3, 8, 15, 29, 45, 47-48)}

Like Slo., Damasio (Dam.) often appears to define the architecture of a CME in terms of cognitive and non-cognitive emotions [15, pp. 72-73], [26, pp. 3-6], [42, pp. 160], [22, pp. 97-98]. This aligns with its idea of two emotion types: innate, evolution-based primary emotions and learned, cognition-driven secondary emotions that trigger the primary system [136, pp. 131-139]. WASABI (8) found Dam. insufficiently specific for formalization, but drew assumptions to make it so [22, pp. 54, 97]. The resulting model is comparable to Sch.. WASABI found similar overlaps with [128], which extends OCC with neurobiological evidence, motivating its combination with Dam..

Two specific features of Dam. are useful for specific CME tasks. One is emotion's influence on decision-making [136, pp. 126, 128], which can drive the design of CME behavior [15, pp. 72], [16, pp. 63], [38, pp. 330]. Directly related to decision-making, the second feature is the Somatic Marker

7. Since Sloman considers the brain to be an information processing system [135, pp. 206-207], it is grouped with the neurobiological theories. 
Hypothesis (SMH), which describes how secondary emotions are learned and connected to the primary emotion system [136, pp. 137, 145, 174]. The SMH has been used as-described to elicit emotions from memories by learned associations between stimuli and emotions [15, pp. 73], and as a clever way to mark different types of inputs with common information to coordinate further functions [55, pp. 133, 151].

Damasio posits that CMEs cannot use this theory because of the biological connection between the mind and body [136, pp. 249-250], suggesting that Dam. cannot be implemented in agents without a physical body. However, it is possible to use a version of SOM that bypasses the body [136, pp. 155-158] as described by WASABI [22, pp. 50, 56].

\subsubsection{LeDoux (Systems 2, 4, 8, 17, 47)}

LeDoux (LD), often alongside Dam., appears in CMEs to define their architecture in terms of cognitive and noncognitive emotions. Damasio and LeDoux applaud each other's work [136, pp. 133], [137, pp. 250, 298]. This could explain their frequent co-use or connection in CMEs [15, pp. 72-73], [164, pp. 1, 5], [22, pp. 161].

LD and Dam. are complementary, agreeing that emotion is critical in decision-making and cannot be separated from the body [137, pp. 41, 176]. Where Dam. focuses on emotional learning and its relation to cognition (i.e. secondary emotions), LD studies the mechanisms of emotional conditioning (i.e. primary emotions) — specifically Fear [137, pp. 127-128]. WASABI (8) drew from this so that the elicitation of Fear did not require reasoning [22, pp. 87].

LD views emotions as biological functions with different neural systems that evolution has maintained across species [137, pp. 106-107, 171], which might have inspired the emotion-to-expression interface in InFra (17) [24, pp. 27]. Each emotion has a mechanism that is programmed to detect innate stimuli relevant to the system's function [137, pp. 134, 143, 161-163, 165, 175-176]. Cognition allows these systems to learn new triggers-which are indistinguishable from one another-and keep the same behavior and speed as innate ones. The ability to unconsciously and quickly perceive and form persistent emotional memories is the brain's most efficient learning and memory functions [137, pp. 182, 266], which FLAME (4) uses for some of its learning processes to improve its adaptability [11, pp. 227, 237-238].

\section{Discussion}

Surveying CMEs and the affective theories used brought out some commonalities. We discuss use themes, computerizing theories, cooperation between perspectives, psychologist influences and other sources of influence.

\subsection{Use themes}

The surveyed CMEs use a variety of theories for different purposes (Table 7). The reasons for choosing particular theories are not always clear. However, there are clear trends in how they use affective theories. Even in CMEs without explicit choice rationale, these uses align with different aspects of the theories. This is indicative of their strengths, which tend to be similar within each perspective.
TABLE 7

Number of Uses of Affective Theories in Surveyed CMEs

\begin{tabular}{lccc|c}
\hline & $\begin{array}{c}\text { Emotion } \\
\text { Generation }\end{array}$ & $\begin{array}{c}\text { Emotion } \\
\text { Expression }\end{array}$ & Other & Total \\
\hline OCC & 39 & 5 & 8 & 52 \\
\hline Ek. & 12 & 9 & - & 21 \\
Sch. & 12 & - & 6 & 18 \\
\hline PAD & 5 & 1 & 10 & 16 \\
\hline Frj. & 6 & 3 & 6 & 15 \\
\hline Laz. & 5 & 7 & 2 & 14 \\
\hline Plu. & 6 & 1 & 2 & 9 \\
\hline V-A & 5 & 2 & 1 & 8 \\
\hline Dam. & 2 & 2 & 4 & 8 \\
\hline Ros. & 6 & - & 1 & 7 \\
\hline O \& JL & 5 & - & 2 & 7 \\
\hline Slo. & 1 & 1 & 5 & 7 \\
Iz. & 3 & 2 & 1 & 6 \\
\hline LD & 4 & - & 2 & 6 \\
\hline S \& K & 4 & - & 1 & 5 \\
\hline \hline
\end{tabular}

The discrete theories appear when types of emotion must be clearly distinguished. This reflects a strength of discrete theories, which build a small set of emotion categories that are supported by empirical evidence [7, pp. 9-10]. Identifying and labeling emotion categories helps delimit them, making it easier to talk about them both formally and informally [73, pp. 286], [6, pp. 353]. The discrete perspective is associated with the most empirical evidence of observed emotion effects to emotions [7, pp. 10]. However, discrete theories do not give many details on emotion generation processes, so they are sometimes combined with another theory or used in hand coded designs (e.g. [61, pp. 324]).

Dimensional theories appear when CMEs need a simple and effective model of affect, as another perspective of emotion categories, and/or as a common space for modeling different affective phenomena and their interactions. Their strength lies in their description of affect in a simple way [4, pp. 97], but it can lose information about an emotional state if its resolution exceeds the named dimensions [103, pp. 172], [6, pp. 353]. This might not be appropriate for all CMEs.

Affective computing uses appraisal theories often [4, pp. 97], [156, pp. 55], appearing more frequently than discrete or dimensional ones. This might be due to its ability to comprehensively represent the complexity of emotion processes, receiving consistent empirical support for their hypothesized mechanisms [73, pp. 281-282]. However, they are based on cognition and CMEs seeking to use these theories must be able to account for it [6, pp. 354]. Most of the CMEs surveyed reference appraisal theories in some way.

The neurobiological theories tend to appear when a $\mathrm{CME}$ wants to distinguish between reactive, non-cognitive and deliberative, cognitive emotion processes. All three theories claim mechanisms for fast, "stupid" reactions and slower, deliberative plans that people collectively call "emotions" [135, pp. 230], [136, pp. 133], [137, pp. 161-165]. 


\subsection{Computerizing Theories}

Once an affective theory is chosen, the challenging task of "programming" it arises. Some theories-Frj., Sch., OCC, O \& JL, and Slo.-were deliberately designed to be computationally tractable [34, pp. 247], [73, pp. 279], [126, pp. 181], [131, pp. 30], [165, pp. 231], while others-Damasio and LeDoux, for example-have argued that their theories cannot be computationally realized [136, pp. 249-250], [137, pp. 41, 176]. Whether or not theorists intended their work to be computerized, designers have found ways to make them work. However, how accurately a CME adheres to a theory and/or observed affective phenomenon tends to be directly proportional to how complex the CME is.

Since "emotions" emerge from interacting components of intelligence, neurobiological theories can produce richer models than most, if not all, affective components [165, pp. 226]. They might also be more plausible than appraisal theories [15, pp. 72-73] and better aligned with current findings [42, pp. 160]. However, accurately modeling these theories requires modeling parts of the brain and body. For many CMEs, this might not be feasible or desirable. There will also be questions about the accuracy of the resulting system due to gaps in our understanding of anatomical structures and functions (although aiming for complete accuracy might not be useful to anyone [156, pp. 60]).

Appraisal theories might be best suited for CMEs as they touch on all components and phases of emotion processing [2, pp. 13]. They are also relatively easy to translate to computational representations due to their rule-based tendencies [1, pp. 225] and comparisons to information processing [156, pp. 59]. Some have integrated neurobiological findings, which increases their scientific merit-but it also increases their complexity. For example, empirical test of Sch. have been relatively successful in predicting different patterns in emotion processes [122, pp. 93, 103, 117-118]. However, it is also very complex, connecting its processes to biological systems like the Autonomic Nervous System (ANS) and memory, and allowing for multiple levels of information processing. This might be why Sch. is favored by Cognitive Architectures and Affective Research CMEs like CLARION (24) and MAMID (6), whose assumptions closely follow Sch.'s [13, pp. 136], [33, pp. 6]. These systems would gladly sacrifice computational efficiency for accuracy because their aim is to study affective phenomena. Depending on how the theory is realized, this complexity can also make it difficult to understand how the CME internals work, effectively turning them into a black-box system [35, pp. 143-144].

Many CMEs focus on agent believability rather than accurately modeling affective phenomenon. Enforcing realism and rational intelligence can be detrimental to their goals [70, pp. 11]. For these systems, computational efficiency is more important as they typically interact with users in real-time, and modularity is essential for testing and debugging. This affords more choice in what theories to use and how to model them. For example, dimensional theories are likely the most amenable to efficiency-focused designs. They have the lowest affective resolution, but they are also considered "universal" and individual points can be labeled if needed [2, pp. 12, 15]. However, since they do not define emotion generation, it is left to the designer to determine how much detail they want to implement.

Defining emotion generation computationally without strict adherence to accuracy leaves room for a number of interesting approaches. There are designs that use metadata [54, pp. 765], [47, pp. 33], [14, pp. 236] and mathematical concepts like Bayesian Networks [50, pp. 204], [19, pp. 108], Markov Models [18, pp. 603], [20, pp. 115], and fuzzy logic [11, pp. 229], [53, pp. 2], [45, pp. 3, 7], [24, pp. 29-30], which can be quite efficient but are not necessarily sound. Other theories — typically appraisal—are also conscripted, but might not be modeled in full [70, pp. 52], [67, pp. 36]. CMEs have also improved their efficiency by considering their target domain's limitations, which might involve fewer emotion categories [19, pp. 109], [39, pp. 2], [12, pp. 58] and appraisal variables [52, pp. 150], while others are able to scale as needed [68], [14, pp. 239], [63, pp. 217], [28, pp. 44], [67, pp. 35], [24, pp. 27]. As these have all found some success in achieving their goals, this further emphasizes that accuracy is not always necessary. This opens up the design space while creating a CME that behaves "well enough" for its intended tasks.

\subsection{Prospective Perspective Cooperation}

CMEs will most often use different theories in combination due to their relative strengths and weaknesses. It is relatively easy to combine theories that share the same perspective because their core assumptions tend to align. For example, Ekman, Izard, and Plutchik agree on the function of at least four primary emotions-Joy/Happiness, Sadness, Anger, and Fear-and their ability to interact to produce what people recognize as other, more complex, emotions [111, pp. 69], [110, pp. 254, 258-259], [114, pp. 200, 204-205]. Similar overlaps exist in the appraisal theories' evaluation dimensions and how they label distinct combinations. The dimensional theories, V-A and PAD, are the most obviously compatible-one could simply layer V-A over the P-A plane directly. By staying within one perspective, a CME design can use the individual strengths of each theory with little worry of conflicting assumptions or views.

Combining theories from multiple perspectives poses a more complex challenge, although often necessary to address all aspects of affect needed in the design. For example, OCC is frequently combined with Ek.-which focuses on automatic, hard-wired appraisals rather than evaluations [146, pp. 51] — to produce facial expressions based on cognitively-evaluated events [19, pp. 109], [21, pp. 253], [25, pp. 66], [42, pp. 155], [97, pp. 740-741]. This connection is presumably due to the OCC's association of characteristically similar "linguistic tokens" with each emotion [126, pp. 1-2, 87-88]. By finding similar words, one can fit the emotions from discrete theories into the OCC structure. However, this relies on subjective interpretations, and even the given lists lack empirical validation [126, pp. 172-176]. More pressingly, emotions of the same name might represent different concepts. Fear and Anger in OCC, as with many appraisal theories, are complex emotions requiring flexible, cognitive evaluations [22, pp. 85, 87]. In contrast, the same emotions in discrete theories are simpler, triggered by inflexible hard-wired systems. While they might be expressed with the same physiological changes, behaviors, 
and expressions, their eliciting mechanisms are not of the same kind [2, pp. 15-16]. Whether or not this distinction is important for a particular CME, it should still be addressed as it affects how accurately the theories are modeled. Similar considerations must be made when attempting to align the dimensional theories with the dimensions of appraisal theories, and locating discrete emotions in dimensional space.

These conceptual mismatches does not mean that there are "correct" and "incorrect" theories, or that they are incompatible, especially considering how they overlap and converge on the role of emotion [2, pp. 10-11, 14-15], [73, pp. 281], [6, pp. 352, 354-355]. Rather, they are different views-perspectives - of a complete system, each focusing on different aspects of the emotion process [119, pp. 259]. A healthy, human emotion system seems to rely on both fast, primary and deliberative, secondary emotions [1, pp. 70]. This idea of two emotion types is present in some affective theories, such as [146, pp. 51], [109, pp. 74], [120, pp. 155], [122, pp. 102], and [166, p. 93], and is also supported by empirical investigations of the brain [136, pp. 136-139], [137, pp. 177-178]. Some CMEs have explicitly modeled these two "pathways", including [16, pp. 63], [15, pp. 73], [42, pp. 160], [22, pp. 98], and [60, pp. 258]. Correspondingly, one way that each perspective could be assigned roles in CME designs to address different aspects of emotion generation is:

- Neurobiological theories provide guidelines for how to unite disparate emotion processing pathways into a coherent system,

- Discrete theories drive the creation of a limited set of fast, hard-wired [6, pp. 366] reactions to specific stimuli ("primary emotions", "low road"),

- Appraisal theories drive the deliberative, slower systems for emotion elicitation that require planning and/or reasoning ("secondary emotions", "high road") that can account for language and sociocultural factors, allowing for a broader range of identifiable emotions, and

- Dimensional theories provide a common space for merging the outcomes of each emotion pathway in the spirit of appraisal registers [122, pp. 105], [166, p. 93] while allowing other types of affect to interact with emotion.

\subsection{Psychologists Directly Involved in CME Design}

Translating a psychological theory into a CME is difficult because it involves formalizing informal concepts and documenting hidden assumptions [9, pp. 22-23]. This process can be harrowing, as one is often left guessing at the theorist's original intent and there might be no way to know for sure. While assumptions are unavoidable, some CMEs can act as secondary sources because originator(s) of the theories used were involved in the design. CMEs designed under the influence of a theory's creator are critical, as the formalization and documentation effort comes from the most accurate information source.

Frijda supervised the development of both ACRES (25) and the Will architecture (27). ACRES is designed as a partial test of its functionality, treating the theory as a design specification [34, pp. 237, 247]. Will-the spiritual successor of ACRES - proposes a reasonable extension of Frj.: emotion, moods, sentiments, and personality are related by focus and duration [36, pp. 135-136, 138]. This is convenient for
CMEs, as it shows that these affective types can share the same underlying structure. Scherer directly influenced the design of ELSA (28). This CME is particularly relevant, as its purpose is to show that Sch.-which takes an information systems view on emotion processes [122, pp. 103] — can be computationally operationalized and used as a research tool [35, pp. 142-143]. Ortony provided direct supervision for AffectR (1) [10, pp. iv], which presumably makes it the most faithful account of OCC emotion generation processes and action tendency taxonomy. More details are in Section 2.3.5.

A number of CMEs have also been indirectly guided by psychologists via other systems [38, pp. 325] or by consultation [38, pp. 332], [17, pp. 281] [37, pp. 303], [167, pp. 89], [22, pp. vii], [145, pp. 101]. These systems provide valuable insights, though their usefulness is limited to specific elements. Caution must be used, as it can be difficult to tell which parts relied on consultation and which did not.

\subsection{Data, Models, and Other Theories}

Affective theories are not self-contained. They do not address all aspects of emotion generation that a CME might need, such as emotion intensity and cognitive organization. In these cases, CMEs might draw on additional affective theories or start to look beyond them to other sources.

A number of systems-like TABASCO (7), FAtiMA (35), AKR (10), SOM (20), HybridC (19), Greta (37), Kismet (47), and MM (59) - strengthen or extend their chosen theoretical foundations by supporting it with other comparable or complementary theories [17, pp. 266], [44, pp. 130, 132], [18, pp. 594, 599-600, 606], [29, pp. 218, 247], [25, pp. 6364], [46, pp. 91], [55, pp. 129], [69, pp. 6, 8-9]. Some, like FLAME (4), cite additional work to support perceived shortcomings in a foundational theory [11, pp. 223, 233-234, 239]. Yet other CMEs use additional sources to connect emotion with other system components, such as mood [26, pp. 5 6], [15, pp. 72], [20, pp. 119] ${ }^{8}$, social variables [65, pp. 288], and emotion expression [24, pp. 27]. An interesting example is POMDP-CA (50), which uses [169] to mathematically define unexpectedness [56, pp. 269], similar to suddenness in Sch. [122, pp. 95]. This is necessary to appraise Surprise in both Ros. [123, pp. 267] and OCC [126, pp. 126]. Three sources in particular have left lasting impressions in affective computing: Picard [1], Minsky [170], and empirical data.

A pioneer of affective computing, Picard offers a computer science-friendly view on affect, offering models and ideas for CME design. AKR (10) references it to justify its use of Markov Models for emotion dynamics [18, pp. 603]. PMES (13) uses Picard to define an emotion intensity function [21, pp. 252]. DER (15) uses Picard to define emotion intensity functions, dynamic filters so that mood influences emotions, and to describe emotional stimulus impulses [26, pp. 7-8]. KaMERo (48) cites Picard for comparable reasons [60, pp. 259-260]. IM-PMEB (14) and FAtiMA (35) use Picard to define an emotion intensity decay function [23, pp. 68], [44, pp. 130-131]. SocioEmo (56) references both Picard and ALMA (38) for this task [65, pp. 289]. Presence (45) cites Picard for their separation of primary and

8. [20, pp. 119] notes that the idea of an activation threshold in [168] is consistent with OCC. 
secondary emotion processing channels, motivating its use of Slo. and Dam. [42, pp. 160]. Greta (37) cites Picard's "tub of water" metaphor, comparable to Plu., for addressing coexistent emotions in its design considerations [46, pp. 99]. TAME (51) uses Picard for defining emotion dynamics as a system response [63, pp. 211].

Minsky offers a model of human intelligence amenable to AI. Since many affective theories-especially appraisal theories-rely on cognition, Minsky's Society of Mind presents a way to model it. O \& JL explicitly draw parallels to it [131, pp. 32, 39]. EmMod (3) cites Minsky as the main inspiration for its architecture, producing complex behaviors via the interactions of many, simple units [16, pp. 63]. Cathexis (2) compares its models of secondary emotions to Minsky $k$-lines, connecting primary emotions to encountered stimuli [15, pp. 73]. HoE (21) also compares its radial dimensions to $k$-lines [30, pp. 149, 152]. It further cites Minsky for its assumption of independent resources that determine emotion states.

Empirical data, the best source for replicating observable phenomena, is also critical in CMEs. It has been used it for: defining degrees of emotion positivity and negativity [157, pp. 4], emotion effects [13, pp. 136], and how mood influences emotion [23, pp. 69]; deriving emotion intensity functions [51, pp. 419]; quantifying the relationship between emotion intensity, desires, and expectations [11, pp. 232], [20, pp. 125]; and gesture models [43, pp. 302]. Of these, only MAMID (6) does not state its source. Some systems have moved to a purely data-driven approaches (e.g. [171], [172]), implying that empirical data will continue to be a big part of CME design.

Even this extension to the prevalent theories in the surveyed CMEs does not cover all the potential affective theory candidates (see [73, pp. 280-281]). However, it does provide a reference for exploring the domain of affective theories and a starting point for thinking about affect in terms of computational models. For example, if one is considering VA then they might look into [173] to see if it fits their needs better. One should not feel limited to what is listed herethere might yet be inspiration in unlikely places (e.g. [174]).

\section{Conclusion}

We have surveyed the ways that CMEs use affective theories. It examines CMEs from different application domains to see how they used the theories for emotion generation, expression, and other design purposes. A comparison of these uses found that each type of affective theory filled a similar role across the application domains:

- Discrete theories define what emotions a CME expresses and how it does so,

- Dimensional theories provide a simple representation that can describe different types of affect in a common space,

- Appraisal theories connect emotion to cognition-using it to drive plans and behaviors-with a finite set of defined evaluations, and

- Neurobiological theories unite the reactionary and deliberative emotion views, tying them to measurable body states.
Like the survey, this list is not meant to be exhaustive. There are likely other ways that one could apply these theories to CME designs. The hope is that both veteran designers and newcomers to the field use this survey as a starting point for new CME designs by providing them with a practical view of some affective theories and existing CMEs to borrow from and build on.

\section{REFERENCES}

[1] R. W. Picard, Affective Computing. Cambridge, MA, USA: MIT Press, 1997.

[2] K. R. Scherer, "Emotion and emotional competence: Conceptual and theoretical issues for modelling agents," in A Blueprint for Affective Computing, ser. Affective Science, K. R. Scherer, T. Bänziger, and E. Roesch, Eds. New York, NY, USA: Oxford Univ. Press, 2010, ch. 1, sec. 1, pp. 3-20.

[3] R. Fathalla, "Emotional models: Types and applications," Int. J. Synthetic Emotions, vol. 11, no. 2, pp. 1-18, Jan.-Jun. 2020

[4] C. Lisetti and E. Hudlicka, "Why and how to build emotionbased agent architectures," in The Oxford Handbook of Affective Computing, R. Calvo, S. D'Mello, J. Gratch, and A. Kappas, Eds. New York, NY, USA: Oxford Univ. Press, Jan. 2015, ch. 8, pp. 94-109.

[5] E. Osuna, L. Rodríguez, J. O. Gutierrez-Garcia, and L. A. Castro, "Development of computational models of emotions: A software engineering perspective," Cogn. Syst. Res., vol. 60, pp. 1-19, May 2020.

[6] J. Broekens, "Emotion," in The Handbook on Socially Interactive Agents: 20 Years of Research on Embodied Conversational Agents, Intelligent Virtual Agents, and Social Robotics Volume 1: Methods, Behavior, Cognition, B. Lugrin, C. Pelachaud, and D. Traum, Eds. New York, NY, USA: ACM, 2021, ch. 10, pp. 349-384.

[7] E. Hudlicka, "From habits to standards: Towards systematic design of emotion models and affective architectures," in Emotion Modeling: Towards Pragmatic Computational Models of Affective Processes, ser. Lecture Notes in Computer Science, T. Bosse, J. Broekens, J. Dias, and J. van der Zwaan, Eds. Cham, Switzerland: Springer Int., Nov. 2014, vol. 8750, pp. 3-23.

[8] L. A. Goodman, "Snowball sampling," Ann. Math. Statist., vol. 32, no. 1, pp. 148-170, Mar. 1961.

[9] S. Marsella, J. Gratch, and P. Petta, "Computational models of emotion," in A Blueprint for Affective Computing, ser. Affective Science, K. R. Scherer, T. Bänziger, and E. Roesch, Eds. New York, NY, USA: Oxford Univ. Press, 2010, ch. 1, sec. 2, pp. 21-41.

[10] C. Elliott, "The affective reasoner: A process model of emotions in a multi-agent system," Ph.D. dissertation, Dept. Comp. Sci., Northwestern Univ., Evanston, IL, USA, 1992.

[11] M. S. El-Nasr, J. Yen, and T. R. Ioerger, "FLAME-Fuzzy logic adaptive model of emotions," Auton. Agents Multi-Agent Syst., vol. 3, no. 3, pp. 219-257, Sep. 2000.

[12] M. A. Salichs and M. Malfaz, "A new approach to modeling emotions and their use on a decision-making system for artificial agents," IEEE Trans. Affective Comput., vol. 3, no. 1, pp. 56-68, Jan.-Mar. 2012.

[13] E. Hudlicka, "Modeling cognition-emotion interactions in symbolic agent architectures: Examples of research and applied models," in Cognitive Architectures, ser. Intelligent Systems, Control, and Automation: Science and Engineering, F. M. Aldinhas, J. Silva Sequeira, and R. Ventura, Eds. Cham, Switzerland: Springer Nature, 2019, vol. 94, pp. 129-143.

[14] H. Prendinger, S. Saeyor, and M. Ishizuka, "MPML and SCREAM: Scripting the bodies and minds of life-like characters," in Life-Like Characters: Tools, Affective Functions, and Applications, ser. Cognitive Technologies, H. Prendinger and M. Ishizuka, Eds. Berlin, Germany: Springer-Verlag, 2004, pp. 213-242.

[15] J. D. Velásquez, "When robots weep: Emotional memories and decision-making," in Proc. 15th Nat. Conf. Artif. Intell. (AAAI-98), Madison, WI, USA, Jul. 26-30, 1998, pp. 70-75.

[16] H. Ushida, Y. Hirayama, and H. Nakajima, "Emotion model for life-like agent and its evaluation," in Proc. 15th Nat. Conf. Artif. Intell. (AAAI-98), Madison, WI, USA, Jul. 26-30, 1998, pp. 62-69.

[17] P. Petta, "The role of emotions in a tractable architecture for situated cognizers," in Emotions in Humans and Artifacts, R. Trappl, P. Petta, and S. Payr, Eds. Cambridge, MA, USA: MIT Press, 2002, ch. 9, pp. 251-288. 
[18] C. L. Lisetti and P. Gmytrasiewicz, "Can a rational agent afford to be affectless? A formal approach," Appl. Artif. Intell., vol. 16, no. 7-8, pp. 577-609, Aug. 2002.

[19] S. Kshirsagar and N. Magnenat-Thalmann, "A multilayer personality model," in Proc. 2nd Int. Symp. Smart Graph. (SMARTGRAPH'02), Hawthorne, NY, USA, Jun. 11-13, 2002, pp. 107-115.

[20] T. Duy Bui, "Creating emotions and facial expressions for embodied agents," Ph.D. dissertation, Dept. Comp. Sci., Univ. Twente, Enschede, Netherlands, 2004.

[21] Y. Guoliang, W. Zhiliang, W. Guojiang, and C. Fengjun, "Affective computing model based on emotional psychology," in Proc. 2nd Int. Conf. Natural Comput.: Adv. Natural Comput. (ICNC 2006), Part 1, ser. Lecture Notes in Computer Science, L. Jiao, L. Wang, X. Gao, J. Liu, and F. Wu, Eds., vol. 4221. Berlin, Germany: Springer-Verlag, Sep. 2006, pp. 251-260.

[22] C. Becker-Asano, "WASABI: Affect simulation for agents with believable interactivity," Ph.D. dissertation, Fac. Technol., Univ. Bielefeld, Bielefeld, Germany, 2008.

[23] M. Shvo, J. Buhmann, and M. Kapadia, "An interdependent model of personality, motivation, emotion, and mood for intelligent virtual agents," in Proc. 19th ACM Int. Conf. Intell. Virtual Agents (IVA'19), Paris, France, Jul. 2-5, 2019, pp. 65-72.

[24] S. Castellanos, L. Rodríguez, L. A. Castro, and J. O. GutierrezGarcia, "A computational model of emotion assessment influenced by cognition in autonomous agents," Biol. Inspired Cogn. Architectures, vol. 25, pp. 26-36, Aug. 2018.

[25] S. Jain and K. Asawa, "Modeling of emotion elicitation conditions for a cognitive-emotive architecture," Cogn. Syst. Res., vol. 55, pp. 60-76, Jun. 2019.

[26] E. Tanguy, J. J. Bryson, and P. J. Willis, "A dynamic emotion representation model within a facial animation system," Dept. Comput. Sci., Univ. Bath, Bath, UK, Tech. Rep. CSBU-2005-14, Nov. 2005.

[27] B. Alfonso, E. Vivancos, and V. Botti, "Toward formal modeling of affective agents in a BDI architecture," ACM Trans. Internet Technol. (TOIT), vol. 17, no. 1, Mar. 2017, Art. no. 5.

[28] J. Dias, S. Mascarenhas, and A. Paiva, "FAtiMA Modular: Towards an agent architecture with a generic appraisal framework," in Emotion Modeling, ser. Lecture Notes in Computer Science, T. Bosse, J. Broekens, J. Dias, and v. J., Eds. Cham, Switzerland: Springer Int., 2014, vol. 8750, pp. 44-56.

[29] T. Yanaru, N. Shirahama, K. Yoshida, and M. Nagamatsu, "An emotion processing system based on fuzzy inference and subjective observations," Inf. Sci., vol. 101, no. 3-4, pp. 217-247, Oct. 1997.

[30] E. Cambria, A. Livingstone, and A. Hussain, "The hourglass of emotions," in Cognitive Behavioural Systems, ser. Lecture Notes in Computer Science, A. Esposito, A. M. Esposito, A. Vinciarelli, R. Hoffmann, and V. C. Müller, Eds. Berlin, Germany: SpringerVerlag, 2012, vol. 7403, pp. 144-157.

[31] J. E. Laird, "Introduction," in The Soar Cognitive Architecture. Cambridge, MA, USA: MIT Press, 2012, ch. 1, pp. 1-25.

[32] S. Franklin, T. Madl, S. D'Mello, and J. Snaider, "LIDA: A systems-level architecture for cognition, emotion, and learning," IEEE Trans. Auton. Mental Develop., vol. 6, no. 1, pp. 19-41, Mar. 2014.

[33] R. Sun, N. Wilson, and M. Lynch, "Emotion: A unified mechanistic interpretation from a cognitive architecture," Cogn. Comput., vol. 8, no. 1, pp. 1-14, Feb. 2016.

[34] N. H. Frijda and J. Swagerman, "Can computers feel? Theory and design of an emotional system," Cogn. Emotion, vol. 1, no. 3, pp. 235-257, Nov. 1987.

[35] B. Meuleman, "Computational modeling of appraisal theory of emotion," Ph.D. dissertation, Fac. Psychol. Educ. Sci., Univ. Geneva, Geneva, Switzerland, 2015.

[36] D. Moffat, "Personality parameters and programs," in Creating Personalities for Synthetic Actors, ser. Lecture Notes in Computer Science, R. Trappl and P. Petta, Eds. Berlin, Germany: SpringerVerlag, 1997, vol. 1195, pp. 120-165.

[37] J. Gratch and S. Marsella, "A domain-independent framework for modeling emotion," Cogn. Syst. Res., vol. 5, no. 4, pp. 269-306, Dec. 2004.

[38] J. Gratch, "Émile: Marshalling passions in training and education," in Proc. 4th Int. Conf. Auton. Agents (AGENTS'00), Barcelona, Spain, Jun. 3-7, 2000, pp. 325-332.

[39] E. Mehdi, P. Nico, D. Julie, and P. Bernard, "Modelling character emotion in an interactive virtual environment," in Proc. AISB
2004 Symp. Lang. Speech Gesture Expressive Characters, Leeds, UK, Mar. 29-Apr. 1, 2004, pp. 20-28.

[40] Š. KoreÏko, B. Sobota, and P. Zemianek, "Jadex/JBdiEmo emotional agents in games with purpose: A feasibility demonstration," Simul. Notes Eur., vol. 26, no. 4, pp. 195-204, Dec. 2016.

[41] H. van Dyke Parunak, R. Bisson, S. Brueckner, R. S. Matthews, and J. A. Sauter, "A model of emotions for situated agents," in Proc. 5th Int. Joint Conf. Auton. Agents and Multiagent Syst. (AAMAS'06), Hakodate, Japan, May 8-12, 2006, pp. 993-995.

[42] E. André, M. Klesen, P. Gebhard, S. Allen, and T. Rist, "Integrating models of personality and emotions into lifelike characters," in Int. Workshop Affect. Interact. (IWAI 1999), ser. Lecture Notes in Artificial Intelligence, A. Paiva, Ed., vol. 1814. Berlin, Germany: Springer-Verlag, 2000, pp. 150-165.

[43] S. C. Marsella, W. L. Johnson, and C. LaBore, "Interactive pedagogical drama," in Proc. 4th Int. Conf. Auton. Agents (AGENTS'00), Barcelona, Spain, Jun. 3-7, 2000, pp. 301-308.

[44] J. Dias and A. Paiva, "Feeling and reasoning: A computational model for emotional characters," in Proc. 12th Portuguese Conf. Artif. Intell. Prog. Artif. Intell. (EPIA 2005), ser. Lecture Notes in Computer Science, C. Bento, A. Cardoso, and G. Dias, Eds., vol. 3808. Berlin, Germany: Springer-Verlag, 2005, pp. 127-140.

[45] H. Jones and N. Sabouret, "TARDIS-A simulation platform with an affective virtual recruiter for job interviews," in Proc. 1st Int. Workshop Intell. Digital Games Empowerment Inclusion (FDG/IDGEI 2013), Chania, Crete, Greece, May 14-17, 2013, [Online]. Available: http://www.fdg2013.org/program/ workshops/papers/IDGEI2013/idgei2013_8.pdf.

[46] F. de Rosis, C. Pelachaud, I. Poggi, V. Carofiglio, and B. De Carolis, "From Greta's mind to her face: Modelling the dynamics of affective states in a conversational embodied agent," Int. J. Human-Comput. Stud., vol. 59, no. 1-2, pp. 81-118, Jul. 2003.

[47] P. Gebhard, "ALMA: A layered model of affect," in Proc. 4th Int. Joint Conf. Auton. Agents Multiagent Syst. (AAMAS '05), Utrecht, The Netherlands, Jul. 25-29, 2005, pp. 29-36.

[48] Z. Kasap, M. B. Moussa, P. Chaudhuri, and N. MagnenatThalmann, "Making them remember-emotional virtual characters with memory," IEEE Comput. Graph. Appl., vol. 29, no. 2, pp. 20-29, Mar.-Apr. 2009.

[49] J. Zhang, J. Zheng, and N. Magnenat-Thalmann, "Modeling personality, mood, and emotions, " in Context Aware Human-Robot and Human-Agent Interaction, ser. Human-Computer Interaction Series, N. Magnenat-Thalmann, J. Yuan, D. Thalmann, and B. You, Eds. Cham, Switzerland: Springer Int., 2016, ch. 10, pp. 211-236.

[50] J. E. Ball and J. Breese, "Emotion and personality in a conversational character," in Embodied Conversational Agents, J. Cassell, J. Sullivan, S. Prevost, and E. F. Churchill, Eds. Cambridge, MA, USA: MIT Press, 2000, ch. 7, pp. 189-219.

[51] M. Ochs, D. Sadek, and C. Pelachaud, "A formal model of emotions for an empathic rational dialog agent," Auton. Agents Multi-Agent Syst., vol. 24, no. 3, pp. 410-440, May 2012.

[52] A. Yacoubi and N. Sabouret, "TEATIME: A formal model of action tendencies in conversational agents," in Proc. 10th Int. Conf. Agents Artif. Intell. (ICAART 2018), vol. 2, Madeira, Portugal, Jan. 16-18, 2018, pp. 143-153.

[53] A. van der Heide and G. Trivino, "Simulating emotional personality in human computer interfaces," in Proc. 2010 IEEE Int. Conf. Fuzzy Syst. (FUZZ-IEEE), Barcelona, Spain, Jul. 18-23, 2010, pp. $1-7$.

[54] Y. Arafa and A. Mamdani, "Virtual personal service assistants: Real-time characters with artificial hearts," in Proc. IEEE Int. Conf. Syst. Man Cybern. (IEEE SMC'99), vol. 1, Tokyo, Japan, Oct. 12-15, 1999, pp. 762-767.

[55] C. Breazeal, "Emotion and sociable humanoid robots," Int. J. Human-Comput. Stud., vol. 59, no. 1-2, pp. 119-155, Jul. 2003.

[56] H.-R. Kim and D.-S. Kwon, "Computational model of emotion generation for human-robot interaction based on the cognitive appraisal theory," J. Intell. Robot. Syst., vol. 60, no. 2, pp. 263-283, Apr. 2010.

[57] N. Masuyama, C. K. Loo, and M. Seera, "Personality affected robotic emotional model with associative memory for humanrobot interaction," Neurocomputing, vol. 272, pp. 213-225, Jan. 2018.

[58] S. Ojha and M.-A. Williams, "Ethically-guided emotional responses for social robots: Should I be angry?" in Int. Conf. Social Robot. (ICSR 2016), ser. Lecture Notes in Computer Science, 
A. Agah, J. Cabibihan, A. Howard, M. Salichs, and H. He, Eds., vol. 9979. Cham, Switzerland: Springer Int., 2016, pp. 233-242.

[59] X. Qi, W. Wang, L. Guo, M. Li, X. Zhang, and R. Wei, "Building a Plutchik's wheel inspired affective model for social robots," J. Bionic Eng., vol. 16, no. 2, pp. 209-221, Mar. 2019.

[60] J.-C. Park, H.-R. Kim, Y.-M. Kim, and D.-S. Kwon, "Robot's individual emotion generation model and action coloring according to the robot's personality," in Proc. 18th IEEE Int. Symp. Robot Human Interact. Commun. (RO-MAN 2009), Toyama, Japan, Sep.27-Oct. 2, 2009, pp. 257-262.

[61] R. Kirby, J. Forlizzi, and R. Simmons, "Affective social robots," Robot. Auton. Syst., vol. 58, no. 3, pp. 322-332, Mar. 2010

[62] T. Dang and D. Duhaut, "Experimentation with GRACE, the generic model of emotions for computational applications," in Proc. 2nd Mediterranean Conf. Intell. Syst. Automat. (CISA'09), L. Beji, S. Otmane, and A. Abichou, Eds., vol. 1107, Zarzis, Tunisia, Mar. 23-25, 2009, pp. 135-140.

[63] L. Moshkina, S. Park, R. C. Arkin, J. K. Lee, and H. Jung, "TAME: Time-varying affective response for humanoid robots," Int. J. Social Robot., vol. 3, no. 3, pp. 207-221, Feb. 2011.

[64] J. Broekens, E. Hudlicka, and R. Bidarra, "Emotional appraisal engines for games," in Emotion in Games: Theory and Praxis, ser. Socio-Affective Computing, K. Karpouzis and G. N. Yannakakis, Eds. Cham, Switzerland: Springer Int., 2016, vol. 4, ch. 13, pp. 215-232.

[65] M. Ochs, N. Sabouret, and V. Corruble, "Simulation of the dynamics of nonplayer characters' emotions and social relations in games," IEEE Trans. Comput. Intell. AI in Games, vol. 1, no. 4, pp. 281-297, Nov. 2009.

[66] R. Bidarra, R. Schaap, and K. Goossens, "Growing on the inside: Soulful characters for video games," in Proc. 2010 IEEE Conf. Comput. Intell. Games (CIG'10), Copenhagen, Denmark, Aug. 1821, 2010, pp. 337-344.

[67] A. Popescu, J. Broekens, and M. Van Someren, "GAMYGDALA An emotion engine for games," IEEE Trans. Affective Comput., vol. 5, no. 1, pp. 32-44, Jan.-Mar. 2014.

[68] J. Broekens and D. DeGroot, "Scalable and flexible appraisal models for virtual agents," in Proc. 5th Game-On Int. Conf. Comput. Games: Artif. Intell., Design, Educ. (CGAIDE'2004), Q. Mehdi, N. Gough, S. Natkin, and D. Al-Dabass, Eds., Reading, UK, Nov. 8-10, 2004, pp. 208-215.

[69] M. P. Eladhari, "The mind module - using an affectand personality computational modelas a game-play element," IEEE Trans. Affective Comput., vol. 5, no. 1, pp. 3-16, Jan.-Mar. 2014.

[70] W. S. N. Reilly, "Believable social and emotional agents," Ph.D. dissertation, Dept. Comp. Sci., Carnegie Mellon Univ., Pittsburgh, PA, USA, 1996.

[71] C. A. Martinho, A. M. Paiva, and M. R. Gomes, "Emotions for a motion: Rapid development of believable pathematic agents in intelligent virtual environments," Appl. Artif. Intell., vol. 14, no. 1, pp. 33-68, Jan. 2000.

[72] B. Grilo, "Emotional agents in a serious game of cooperation and competition," Master's thesis, Dept. Comput. Eng., Univ. Lisbon, Lisbon, Portugal, 2015.

[73] K. R. Scherer, "Towards a prediction and data driven computational process model of emotion," IEEE Trans. Affective Comput., vol. 12, no. 2, pp. 279-292, Apr.-Jun. 2021.

[74] R. P. Bonasso, R. J. Firby, E. Gat, D. Kortenkamp, D. P. Miller, and M. G. Slack, "Experiences with an architecture for intelligent, reactive agents," J. Exp. Theor. Artif. Intell., vol. 9, no. 2-3, pp. 237256, Apr. 1997.

[75] C. Becker, S. Kopp, and I. Wachsmuth, "Simulating the emotion dynamics of a multimodal conversational agent," in Proc. Tut. Res. Workshop on Affect. Dialogue Syst. (ADS 2004), ser. Lecture Notes in Computer Science, E. André, L. Dybkjær, W. Minker, and P. Heisterkamp, Eds., vol. 3068. Berlin, Germany: SpringerVerlag, 2004, pp. 154-165.

[76] C. Castelfranchi, F. de Rosis, and R. Falcone, "Social attitudes and personalities in agents," in "Proc. AAAI Fall Symp. Socially Intell. Agents," AAAI, Menlo Park, CA, USA, Tech. Rep. FS-97-02, pp. 16-21, Nov. 1997.

[77] N. Magnenat-Thalmann and S. Kshirsagar, "Communicating with autonomous virtual humans," in presented at the 17th TWENTE Workshop Lang. Technol., Enschede, Netherlands, Oct. 18-20, 2000.

[78] B. Alfonso, E. Vivancos, and V. J. Botti, "An open architecture for affective traits in a BDI agent," in Proc. 6th Int. Conf. Evol. Comput.
Theory Appl. (ECTA 2014), A. Rosa, J. J. Merelo, and J. Filipe, Eds., vol. 1, Rome, Italy, Oct. 22-24, 2014, pp. 320-325.

[79] R. Vieira, Á. F. Moreira, M. Wooldridge, and R. H. Bordini, "On the formal semantics of speech-act based communication in an agent-oriented programming language," J. Artif. Intell. Res., vol. 29, pp. 221-267, Jun. 2007.

[80] M. Y. Lim, J. Dias, R. Aylett, and A. Paiva, "Creating adaptive affective autonomous NPCs," Auton. Agents Multi-Agent Syst., vol. 24, no. 2, pp. 287-311, Mar. 2012.

[81] S. Jain and K. Asawa, "EMIA: Emotion model for intelligent agent," J. Intell. Syst., vol. 24, no. 4, pp. 449-465, Oct. 2015.

[82] A. Newell, Unified Theories of Cognition. Cambridge, MA, USA: Harvard Univ. Press, 1990.

[83] L. Beaudoin, "Goal processing in autonomous agents," Ph.D. dissertation, School Comput. Sci., Univ. Birmingham, Birmingham, England, 1994.

[84] J. Rickel and W. L. Johnson, "Animated agents for procedural training in virtual reality: Perception, cognition, and motor control," Appl. Artif. Intell., vol. 13, no. 4-5, pp. 343-382, Jun. 1999.

[85] J. E. Cahn, "Generating expression in synthesized speech," Ph.D dissertation, MIT Media Lab, Massachusetts Inst. Technol., Cambridge, USA, 1989.

[86] A. Egges, S. Kshirsagar, and N. Magnenat-Thalmann, "Generic personality and emotion simulation for conversational agents," Comput. Animation Virtual Worlds, vol. 15, no. 1, pp. 1-13, Mar 2004.

[87] A. Pokahr, L. Braubach, and W. Lamersdorf, "Jadex: A BDI reasoning engine," in Multi-Agent Programming, ser. Multiagent Systems, Artificial Societies, and Simulated Organizations (International Book Series), R. H. Bordini, M. Dastani, J. Dix, and A. El Fallah Seghrouchni, Eds. Boston, MA, USA: Springer Science+Business Media, 2005, vol. 15, ch. 6, pp. 149-174.

[88] M. Lauren and R. Stephen, "Map-aware non-uniform automata (MANA) - a New Zealand approach to scenario modelling," $J$. Battlefield Technol., vol. 5, pp. 27-31, Mar. 2002.

[89] R. S. Aylett, S. Louchart, J. Dias, A. Paiva, and M. Vala, "FearNot!-An experiment in emergent narrative," in Proc. 5th Int. Work. Conf. Intell. Virtual Agents (IVA 2005), ser. Lecture Notes in Computer Science, T. Panayiotopoulos, J. Gratch, R. Aylett, D. Ballin, P. Olivier, and T. Rist, Eds., vol. 3661. Berlin, Germany: Springer-Verlag, 2005, pp. 305-316.

[90] P. Gebhard, M. Kipp, M. Klesen, and T. Rist, "Adding the emotional dimension to scripting character dialogues," in Proc. 4th Int. Workshop Intell. Virtual Agents (IVA 2003), ser. Lecture Notes in Computer Science, T. Rist, R. S. Aylett, D. Ballin, and J. Rickel, Eds., vol. 2792. Berlin, Germany: Springer-Verlag, 2003, pp. 4856.

[91] P. Gebhard, M. Klesen, and T. Rist, "Coloring multi-character conversations through the expression of emotions," in Proc. Tut. Res. Workshop Affect. Dialogue Syst. (ADS 2004), ser. Lecture Notes in Computer Science, E. André, L. Dybkjær, W. Minker, and P. Heisterkamp, Eds., vol. 3068. Berlin, Germany: SpringerVerlag, 2004, pp. 128-141.

[92] M. Ochs, C. Pelachaud, and D. Sadek, "Emotion elicitation in an empathic virtual dialog agent," in Pro. Eur. Cogn. Sci. Conf. (EurCogSci07), S. Vosniadou, D. Kayser, and A. Protopapas, Eds., Delphi, Greece, May 23-27, 2007, pp. 452-457.

[93] E. André, T. Rist, and J. Muller, "Employing AI methods to control the behavior of animated interface agents," Appl. Artif. Intell., vol. 13, no. 4-5, pp. 415-448, May 1999.

[94] Y. Kim, H.-E. Lee, K.-H. Park, and Z. Z. Bien, "Steward robot: Emotional agent for subtle human-robot interaction," in Proc. 15th IEEE Int. Symp. Robot Human Interact. Commun. (RO-MAN 2006), Hatfield, UK, Sep. 6-8, 2006, pp. 263-268.

[95] Y.-M. Kim, J.-C. Park, and D.-S. Kwon, "Behavior coordination of socially interactive robot using sentiment relation model," in Proc. 16th IEEE Int. Symp. Robot Human Interact. Commun. (ROMAN 2007), Jeju, Korea (South), Aug. 26-29, 2007, pp. 1034-1039.

[96] A. Bruce, I. Nourbakhsh, and R. Simmons, "The role of expressiveness and attention in human-robot interaction," in Proc. 2002 IEEE Int. Conf. Robot. Automat. (ICRA'02), vol. 4, Washington, DC, USA, May 11-15, 2002, pp. 4138-4142.

[97] N. Masuyama, M. N. Islam, M. Seera, and C. K. Loo, “Application of emotion affected associative memory based on mood congruency effects for a humanoid," Neural Comput. Appl., vol. 28, no. 4, pp. 737-752, Apr. 2017. 
[98] M.-J. Han, C.-H. Lin, and K.-T. Song, "Robotic emotional expression generation based on mood transition and personality model," IEEE Trans. Cybern., vol. 43, no. 4, pp. 1290-1303, Aug. 2013.

[99] J. E. Steephen, "HED: A computational model of affective adaptation and emotion dynamics," IEEE Trans. Affective Comput., vol. 4, no. 2, pp. 197-210, Apr.-Jun. 2013.

[100] C. Katsimerou, I. Heynderickx, and J. Redi, "Predicting mood from punctual emotion annotations on videos," IEEE Trans. Affective Comput., vol. 6, no. 2, pp. 179-192, Apr.-Jun. 2015.

[101] S. Saint-Aimé, B. Le-Pevedic, D. Duhaut, and T. Shibata, "EmotiRob: Companion robot project," in Proc. 16th IEEE Int. Symp. Robot Human Interact. Commun. (RO-MAN 2007), Jeju, Korea (South), Aug. 26-29, 2007, pp. 919-924.

[102] K. Sehaba, N. Sabouret, and V. Corruble, "An emotional model for synthetic characters with personality," in Int. Conf. Affective Comput. Intell. Interact., ser. Lecture Notes in Computer Science, A. C. R. Paiva, R. Prada, and R. W. Picard, Eds., vol. 4738. Berlin, Germany: Springer-Verlag, 2007, pp. 749-750.

[103] R. Schaap and R. Bidarra, "Towards emotional characters in computer games," in Proc. 7th Int. Conf. Entertainment Comput. (ICEC 2008), ser. Lecture Notes in Computer Science, S. M. Stevens and S. Saldamarco, Eds., vol. 5309. Berlin, Germany: Springer, 2008, pp. 167-172.

[104] J. Bates, A. B. Loyall, and W. S. Reilly, "An architecture for action, emotion, and social behavior," in Proc. 4th Eur. Workshop Artif. Social Syst. Eur. Workshop Model. Auton. Agents Multi-Agent World (MAAMAW'92), ser. Lecture Notes in Computer Science (Lecture Notes in Artificial Intelligence), C. Castelfranchi and E. Werner, Eds., vol. 830. Berlin, Germany: Springer-Verlag, 1992, pp. 55-68.

[105] A. B. Loyall, "Believable agents: Building interactive personalities," Ph.D. dissertation, Comput. Sci. Dept., Carnegie Mellon Univ., Pittsburgh, PA, USA, 1997.

[106] B. Antunes, "Believable synthetic characters with social identity," Master's thesis, Univ. Lisbon, Univ. Lisbon, Lisbon, Portugal, 2012.

[107] C. F. Pimentel, "Grateful agents and agents that hold a grudge," in Proc. 5th Int. Conf. Agents Artif. Intell. (ICAART-2013), J. Filipe and A. Fred, Eds., vol. 1, Barcelona, Spain, Feb. 15-18, 2013, pp. 324-329.

[108] C. E. Izard, D. Z. Libero, P. Putnam, and O. M. Haynes, "Stability of emotion experiences and their relations to traits of personality," J. Personality Social Psychol., vol. 64, no. 5, pp. 847-860, May 1993.

[109] C. E. Izard, "Four systems for emotion activation: Cognitive and noncognitive processes," Psychol. Rev., vol. 100, no. 1, pp. 68-90, Jan. 1993.

[110] C. E. Izard and B. P. Ackerman, "Motivational, organizational, and regulatory functions of discrete emotions," in Handbook of Emotions, 2nd ed., M. Lewis and J. M. Haviland-Jones, Eds. New York, NY, USA: Guilford, May 2000, ch. 16, pp. 253-264.

[111] P. Ekman, Emotions Revealed: Recognizing Faces and Feelings to Improve Communication and Emotional Life, 2nd ed. New York, NY, USA: St. Martin's, 2007.

[112] P. Ekman, W. V. Friesen, and J. C. Hager, "Facial Action Coding System: The Manual on CD ROM," 2nd ed., Salt Lake City, UT, USA: A Human Face, 2002 [CD-ROM].

[113] R. Plutchik, Emotion: A Psychoevolutionary Synthesis. New York, NY, USA: Harper \& Row, 1980.

[114] — "Emotions: A general psychoevolutionary theory," in Approaches to Emotion, K. R. Scherer and P. Ekman, Eds. Hillsdale, NJ, USA: L. Erlbaum Associates, 1984, ch. 8, pp. 197-219.

[115] T. Brosch and A. Moors, "Valence," in Oxford Companion to Emotion and the Affective Sciences, D. Sander and K. Scherer, Eds. New York, NY, USA: Oxford Univ. Press, 2009, pp. 401-402.

[116] D. C. Fowles, "Arousal," in Oxford Companion to Emotion and the Affective Sciences, D. Sander and K. Scherer, Eds. New York, NY, USA: Oxford Univ. Press, 2009, p. 50.

[117] W. Wundt, An Introduction to Psychology. New York, NY, USA: Macmillan, 1912.

[118] A. Mehrabian, "Pleasure-Arousal-Dominance: A general framework for describing and measuring individual differences in temperament," Current Psychol., vol. 14, no. 4, pp. 261-292, Dec. 1996.

[119] N. H. Frijda, The Emotions. Cambridge, UK: Cambridge Univ. Press, 1986.
[120] N. H. Frijda and M. Zeelenberg, "Appraisal: What is the dependent?" in Appraisal Processes in Emotion: Theory, Methods, Reserach, ser. Series in Affective Science, K. R. Scherer, A. Schorr, and T. Johnstone, Eds. New York, NY, USA: Oxford Univ. Press, Jan. 2001, ch. 7, pp. 141-155.

[121] R. S. Lazarus, Emotion and Adaptation. New York, NY, USA: Oxford Univ. Press, 1991.

[122] K. R. Scherer, "Appraisal considered as a process of multi-level sequential checking," in Appraisal Processes in Emotion: Theory, Methods, Research, ser. Series in Affective Science, K. R. Scherer, A. Schorr, and T. Johnstone, Eds. New York, NY, USA: Oxford Univ. Press, 2001, ch. 5, pp. 92-120.

[123] I. J. Roseman, A. A. Antoniou, and P. E. Jose, "Appraisal determinants of emotions: Constructing a more accurate and comprehensive theory," Cogn. Emotion, vol. 10, no. 3, pp. 241-278, May 1996.

[124] I. J. Roseman, "Emotional behaviors, emotivational goals, emotion strategies: Multiple levels of organization integrate variable and consistent responses," Emotion Rev., vol. 3, no. 4, pp. 434-443, Sep. 2011.

[125] _ "Functions of anger in the emotion system," in The Function of Emotions: When and Why Emotions Help Us, H. C. Lench, Ed. Cham, Switzerland: Springer Int., 2018, ch. 8, pp. 141-173.

[126] A. Ortony, G. L. Clore, and A. Collins, The Cognitive Structure of Emotions. New York, NY, USA: Cambridge Univ. Press, 1988.

[127] A. Ortony, "On making believable emotional agents believable," in Emotions in Humans and Artifacts, R. Trappl, P. Petta, and S. Payr, Eds. Cambridge, MA, USA: MIT Press, 2002, ch. 6, pp. 189-211.

[128] A. Ortony, D. A. Norman, and W. Revelle, "Affect and protoaffect in effective functioning," in Who Needs Emotions? The Brain Meets the Robot, ser. Series in Affective Science, J. Fellous and M. A. Arbib, Eds. New York, NY, USA: Oxford Univ. Press, 2005, ch. 7, pp. 173-202.

[129] C. A. Smith, L. A. Griner, L. D. Kirby, and H. S. Scott, "Toward a process model of appraisal in emotion," in Proc. 9th Conf. Int. Soc. Res. Emotion (ISRE'96), N. H. Frijda, Ed., Toronto, Canada, 1996, pp. 101-105.

[130] C. A. Smith and L. D. Kirby, "Toward delivering on the promise of appraisal theory," in Appraisal Processes in Emotion: Theory, Methods, Research, ser. Series in Affective Science, K. R. Scherer, A. Schorr, and T. Johnstone, Eds. New York, NY, USA: Oxford Univ. Press, 2001, ch. 6, pp. 121-138.

[131] K. Oatley and P. N. Johnson-Laird, "Towards a cognitive theory of emotions," Cogn. Emotion, vol. 1, no. 1, pp. 29-50, Jun. 1987.

[132] P. N. Johnson-Laird and K. Oatley, "Basic emotions, rationality, and folk theory," Cogn. Emotion, vol. 6, no. 3-4, pp. 201-223, 1992.

[133] K. Oatley, Best Laid Schemes: The Psychology of the Emotions. New York, NY, USA: Cambridge Univ. Press, 1992.

[134] — "The sentiments and beliefs of distributed cognition," in Emotions and Beliefs: How Feelings Influence Thoughts, N. H. Frijda, A. S. R. Manstead, and S. Bem, Eds. Cambridge, UK: Cambridge Univ. Press, 2000, ch. 4, pp. 78-107.

[135] A. Sloman, R. Chrisley, and M. Scheutz, "The architectural basis of affective states and processes," in Who Needs Emotions? The Brain Meets the Robot, ser. Series in Affective Science, J.-M. Fellous and M. A. Arbib, Eds. New York, NY, USA: Oxford Univ. Press, 2005, ch. 8, pp. 203-244.

[136] A. R. Damasio, Descartes' Error: Emotion, Reason, and the Human Brain. New York, NY, USA: Avon, 1995.

[137] J. E. LeDoux, The Emotional Brain: The Mysterious Underpinnings of Emotional Life, reprint ed. New York, NY, USA: Simon and Schuster, 1998.

[138] J. E. Laird and R. P. Marinier III, "Emotion," in The Soar Cognitive Architecture. Cambridge, MA, USA: MIT Press, 2012, ch. 11, pp. 271-285.

[139] B. R. Steunebrink, M. Dastani, and J. C. Meyer, "The OCC model revisited," in Proc. 4th Workshop Emotion Comput.-Current Res. Future Impact, D. Reichardt, Ed., Paderborn, Germany, Sep. 15, 2009.

[140] E. Hudlicka, "Computational analytical framework for affective modeling: Towards guidelines for designing computational models of emotions," in Handbook of Research on Synthesizing Human Emotion in Intelligent Systems and Robotics, J. Vallverdú, Ed. Hershey, PA, USA: IGI Global, 2014, ch. 1, pp. 1-62. 
[141] C. E. Izard, Human emotions, ser. Emotions, Personality, and Psychotherapy, C. E. Izard and J. L. Singer, Eds. New York, NY, USA: Plenum, 1977.

[142] $(M A X)$, revised ed. Newark, DE, USA: Instructional Resour. Center, Univ. of Delaware, 1983.

[143] N. A. Remington, L. R. Fabrigar, and P. S. Visser, "Reexamining the circumplex model of affect," J. Personality Social Psychol., vol. 79, no. 2, pp. 286-300, Aug. 2000.

[144] R. Plutchik and H. R. Conte, "Circumplex models of personality and emotions," in Circumplex Models of Personality and Emotions, R. Plutchik and H. R. Conte, Eds. Washington, DC, USA: American Psychological Association, 1997, ch. Introduction, pp. $1-14$.

[145] Y. Susanto, A. G. Livingstone, B. C. Ng, and E. Cambria, "The hourglass model revisited," IEEE Intelligent Systems, vol. 35, no. 5, pp. 96-102, Sep.-Oct. 2020.

[146] P. Ekman, "Basic emotions," in Handbook of Cognition and Emotion, T. Dalgleish and M. Power, Eds. New York, NY, USA: John Wiley \& Sons, 1999, ch. 3, pp. 45-60.

[147] L. F. Barrett and E. Bliss-Moreau, "Affect as a psychological primitive," in Advances in Experimental Social Psychology, M. P. Zanna, Ed. New York, NY, USA: Academic, 2009, vol. 41, ch. 4, pp. 167-218.

[148] R. Reisenzein, "Pleasure-arousal theory and the intensity of emotions," J. Personality Social Psychol., vol. 67, no. 3, p. 525, 1994.

[149] N. H. Frijda, A. Ortony, J. Sonnemans, and G. L. Clore, "The complexity of intensity: Issues concerning the structure of emotion intensity," in Emotion: Review of Personality and Social Psychology, M. S. Clark, Ed. Newbury Park, CA, USA: Sage, 1992, ch. 3, pp. 60-89.

[150] P. Gebhard and K. H. Kipp, "Are computer-generated emotions and moods plausible to humans?" in Proc. 6th Int. Conf. Intell. Virtual Agents (IVA 2006), ser. Lecture Notes in Computer Science, J. Gratch, M. Young, R. Aylett, D. Ballin, and P. Olivier, Eds., vol. 4133. Berlin, Germany: Springer-Verlag, 2006, pp. 343-356.

[151] P. T. Costa and R. R. McCrae, "Normal personality assessment in clinical practice: The NEO Personality Inventory," Psychol. Assessment, vol. 4, no. 1, pp. 5-13, Mar. 1992.

[152] R. Reisenzein, E. Hudlicka, M. Dastani, J. Gratch, K. Hindriks, E. Lorini, and J. C. Meyer, "Computational modeling of emotion: Toward improving the inter-and intradisciplinary exchange," IEEE Trans. Affective Comput., vol. 4, no. 3, pp. 246-266, May 2013.

[153] C. A. Smith and R. S. Lazarus, "Emotion and adaptation," in Handbook of Personality: Theory and Research, L. A. Pervin, Ed. New York, NY, USA: Guilford, 1990, ch. 23, pp. 609-637.

[154] S. Marsella, J. Gratch, and J. Rickel, “Expressive behaviors for virtual worlds," in Life-Like Characters, ser. Cognitive Technologies, H. Prendinger and M. Ishizuka, Eds. Berlin, Germany: SpringerVerlag, 2004, pp. 317-360.

[155] D. Traum, W. Swartout, S. Marsella, and J. Gratch, "Fight, flight, or negotiate: Believable strategies for conversing under crisis," in Proc. 5th Int. Work. Conf. Intell. Virtual Agents (IVA 2005), ser. Lecture Notes in Computer Science, T. Panayiotopoulos, J. Gratch, R. Aylett, D. Ballin, P. Olivier, and T. Rist, Eds., vol. 3661. Berlin, Germany: Springer-Verlag, 2005, pp. 52-64.

[156] S. Marsella and J. Gratch, "Appraisal models," in The Oxford Handbook of Affective Computing, R. Calvo, S. D'Mello, J. Gratch, and A. Kappas, Eds. Oxford Univ. Press, Jan. 2015, ch. 5, pp. 94-109.

[157] S. Ojha and M.-A. Williams, "Emotional appraisal: A computational perspective," in Proc. 5th Annu. Conf. Adv. Cogn. Syst., Troy, NY, USA, May 12-14, 2017, pp. 1-15.

[158] K. R. Scherer, "Psychological models of emotion," in The Neuropsychology of Emotion, ser. Series in Affective Science, J. C. Borod, Ed. New York, NY, USA: Oxford Univ. Press, 2000, ch. 6, pp. 137-162.

[159] C. Bartneck, "Integrating the OCC model of emotions in embodied characters," in Proc. Workshop Virtual Conversational Characters (VCC): Appl. Methods Res. Challenges, Melbourne, Australia, Nov. 29, 2002.

[160] B. Irfan, A. Narayanan, and J. Kennedy, "Dynamic emotional language adaptation in multiparty interactions with agents," in Proc. 20th ACM Int. Conf. Intell. Virtual Agents (IVA'20), Scotland, UK (Virtual Event), Oct. 20-22, 2020, Art. no. 27.
[161] M. Kipp, T. Dackweiler, and P. Gebhard, "Designing emotions: An empirical approach to realistic affect simulation," KIKünstliche Intelligenz, vol. 25, no. 3, pp. 205-211, May 2011.

[162] S. Ojha, M. Williams, and B. Johnston, "The essence of ethical reasoning in robot-emotion processing," Int. J. Social Robot., vol. 10, no. 2, pp. 211-223, Apr. 2018.

[163] L. Rodríguez and F. Ramos, "Computational models of emotions for autonomous agents: Major challenges," Artif. Intell. Rev., vol. 43, no. 3, pp. 437-465, Mar. 2015.

[164] C. Breazeal and J. Velásquez, "Toward teaching a robot 'infant' using emotive communication acts," in Socially Situated Intell.: Workshop held at $S A B^{\prime} 98$, B. Edmonds and K. Dautenhahn, Eds., Zürich, Switzerland, Aug. 21, 1998, [Online]. Available: http:// cfpm.org/cpmrep40.html.

[165] A. Sloman, "Motives, mechanisms, and emotions," Cogn. Emotion, vol. 1, no. 3, pp. 217-233, Nov. 1987.

[166] C. A. Smith and L. D. Kirby, "Consequences require antecedents: Toward a process model of emotion elicitation," in Feeling and Thinking: The Role of Affect in Social Cognition, ser. Studies in Emotion and Social Interaction. Second Series, J. P. Forgas, Ed. New York, NY, USA: Cambridge Univ. Press, 2000, ch. 4, pp. 83106.

[167] S. C. Marsella and J. Gratch, "EMA: A process model of appraisal dynamics," Cogn. Syst. Res., vol. 10, no. 1, pp. 70-90, Mar. 2009.

[168] J. Panksepp, "Basic emotions ramify widely in the brain, yielding many concepts that cannot be distinguished unambiguously... yet," in The Nature of Emotion: Fundamental Questions, ser. Series in Affective Science, P. Ekman and R. J. Davidson, Eds. New York, NY, USA: Oxford Univ. Press, 1994, ch. 2, sec. 7, pp. 86-88.

[169] W. Weaver, "Probability, rarity, interest, and surprise," Sci. Monthly, vol. 67, no. 6, pp. 390-392, Dec. 1948.

[170] M. Minsky, Society of Mind. New York, NY, USA: Simon and Schuster, 1986.

[171] S. Ojha, J. Vitale, S. A. Raza, R. Billingsley, and M.-A. Williams, "Integrating personality and mood with agent emotions," in Proc. 18th Int. Conf. Auton. Agents MultiAgent Syst. (AAMAS'19), Montréal, QC, Canada, May 13-17, 2019, pp. 2147-2149.

[172] Z. Bai, N. Yao, N. Mishra, H. Chen, H. Wang, and N. Magnenat Thalmann, "Enhancing emotional experience by building emotional virtual characters in vr volleyball games," Comput. Animation and Virtual Worlds, vol. 32, no. 3-4, Jun.-Jul. 2021, Art. no. e2008.

[173] J. A. Russell, “A circumplex model of affect," J. Personality Social Psychol., vol. 39, no. 6, pp. 1161-1178, Dec. 1980.

[174] S. N. Nallaperuma and A. S. Karunananda, "EME: An emergent model of emotions," in Proc. 2011 Int. Conf. Adv. ICT Emerg. Regions (ICTer), Colombo, Sri Lanka, Sep. 1-2, 2011, pp. 19-25.

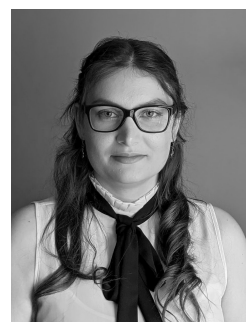

Geneva M. Smith received the M.A.Sc. in software engineering from McMaster University, Hamilton, ON, Canada, in 2017. She is working on her dissertation at the G-ScalE lab in the Department of Computing and Software at McMaster. Her main research focus is computational models of emotions for improving the believability of digital game characters.

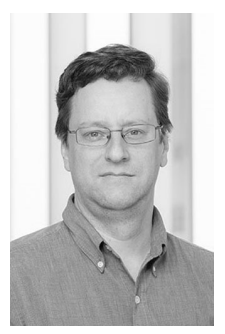

Jacques Carette received his Ph.D. in mathematics from the Université de Paris-Sud, Orsay, France in 1997

$\mathrm{He}$ is currently working as an Associate Professor and the principal investigator of the GScalE Lab in the Department of Computing and Software at McMaster University, Hamilton, ON, Canada. His research interests include game design from a computer science perspective, generative and meta programming, program transformation, and mechanized mathematics. 